\title{
Diversity and evolution of cytochromes P450 in Stramenopiles
}

\author{
Linhong Teng ${ }^{1,2}$, Xiao Fan ${ }^{1}$, David R. Nelson ${ }^{3}$, Wentao Han ${ }^{1}$, Xiaowen Zhang ${ }^{1}$, Dong Xu ${ }^{1}$, Hugues Renault ${ }^{4}$, Gabriel V. \\ Markov $^{5}$, Naihao $\mathrm{Ye}^{1,2, *}$ \\ ${ }^{1}$ Yellow Sea Fisheries Research Institute, Chinese Academy of Fishery Sciences, Qingdao, China, 266071 \\ ${ }^{2}$ Function Laboratory for Marine Fisheries Science and Food Production Processes, Qingdao National \\ Laboratory for Marine Science and Technology, China, 266071 \\ ${ }^{3}$ Department of Microbiology, Immunology and Biochemistry, University of Tennessee Health Science Center, \\ 858 Madison Ave. Suite G01, Memphis, TN, USA 38163 \\ ${ }^{4}$ Institute of Plant Molecular Biology, CNRS, University of Strasbourg, 67084 Strasbourg, France \\ ${ }^{5}$ Sorbonne Université, CNRS, Integrative Biology of Marine Models (LBI2M), Station Biologique de Roscoff \\ (SBR), 29680 Roscoff, France
}

*Author for correspondence: Naihao Ye, Yellow Sea Fisheries Research Institute, Chinese Academy of Fishery Sciences, Qingdao, China, Tel/Fax: +86-532-85830360, E-mail: yenh@ysfri.ac.cn;

\section{Main conclusion}

Comparative genomic analysis of cytochromes $\mathrm{P} 450$ revealed high diversification and dynamic changes in stramenopiles, associated with transcriptional responsiveness to various environment stimuli.

\begin{abstract}
Comparative genomic and molecular evolution approaches were used to characterize P450 diversity in stramenopiles. Phylogenetic analysis further supported the high diversity of P450 in stramenopiles. Three clans were defined, that is, CYP51 clan and CYP97 clan present in brown algae, diatoms and Nannochloropsis gaditana, CYP5014 clan present in oomycetes. Two ancient families, CYP51 and CYP97 showed a close relationship with red algae, green algae and higher plants. Gene gain and loss patterns revealed that six CYP families - CYP51, CYP97, CYP5160, CYP5021, CYP5022, and CYP5165 predated the split of brown algae and diatoms. After they diverged, diatoms gained more CYP families, especially in the cold-adapted species Fragilariopsis cylindrus, in which eight new CYP families were found. Selection analysis revealed that the expanded CYP51 family in the brown alga Cladosiphon okamuranus exhibited a more relaxed selection constraint compared with those of other brown algae and diatoms. Our RNA-seq data further evidenced that most of P450s in Saccharina japonica are highly expressed in large sporophytes, which could potentially promote the large kelp formation in this life stage. Analysis of Ectocarpus siliculosus and diatom microarray transcriptomes showed that many P450s are responsive to stress, limited nutrient or light quality, suggesting their pivotal roles in detoxification or metabolic processes under adverse environmental stimuli. The information provided in this study will be helpful in designing functional experiments and interpreting P450 roles in this particular lineage.
\end{abstract}

Keywords: Cytochrome P450; Comparative genomics; Molecular evolution; Stramenopiles

\section{Introduction}

Cytochromes P450 (P450, CYP) are monooxygenase enzymes found in all life kingdoms, from prokaryotes to eukaryotes, which catalyze a broad range of irreversible, often ratelimiting reactions within metabolic pathways (Nelson 2017). They are one of the largest enzyme superfamilies, showing singular patterns of evolution, including diversification bursts (Nelson 2017). All extant P450s were known to originate from a common ancestral gene, and evolved into diverse forms having different activities (Omura and Gotoh 2017). P450s are involved in the biosynthesis of important specialized metabolites (e.g. sterols, pigments, antioxidants), which are thought to convey adaptive advantages in specific ecological niches. Furthermore, they participate in the detoxification of xenobiotics such as pesticides (Yu et al. 2015). Considering their catalytic diversity, CYPs also offer prospect for potential 
applications in medicine, agriculture and industry through metabolic engineering approaches (Syed et al. 2013; Renault et al. 2014).

The comprehensive repertory of $C Y P$ genes in the genome of a defined species is called the CYPome. Nowadays, with the breadth of sequencing projects, more than 350,000 P450 sequences are available, over 41,000 of which are named, although the functions of the majority are still unknown (Nelson 2017). Nomenclature of CYPs is based on amino acid sequence identity. Generally, sequences having more than $40 \%$ identity belong to one family, and sequences having more than 55\% identity belong to one subfamily. CYP families ranging from 1 to 49, 301 to 499 and from 3001 to 4999 are for animals, 51 to 69, 501 to 699 and 5001 to 6999 for lower eukaryotes, 71 to 99,701 to 999 and 7001 to 9999 for plants, and 101 to 299 and 1001 to 2999 for bacteria. (Nelson 1998; Phillips and Shephard 1998). Ultimately, family assignment is validated by phylogenetic reconstructions. CYP clans are higher classification units. They are deep branching clades on phylogenetic trees that form natural divisions among CYP sequences from one kingdom. Animals have 12 clans and multicellular land plants have 12 too. Clans for algae are not well defined yet (Nelson 2017).

Stramenopiles (also referred to as heterokonts), together with alveolates and rhizarians are called 'SAR clade'(Keeling 2013). The ancestral plastid in this group was derived from red algae via secondary or higher endosymbiosis events, in an organism that may have possessed a green algal plastid (Frommolt et al. 2008; Moustafa et al. 2009) ; Dorrell et al., 2017). The photosynthetic members of the stramenopiles, termed the ochrophytes, are the most diverse algal group with plastids of algal origin (Dorrell et al. 2017). The best characterized ochrophytes, diatoms and multicellular brown algae, constitute two important groups of stramenopiles (Ševčíková et al. 2015; Aleoshin et al. 2016). Diatoms are major primary producers in the ocean (Mock et al. 2017), while submarine kelp forests are the largest marine communities, which serve as spawning grounds for marine animals, as well as being important components for future renewable energy resources (Ye et al. 2015; Teng et al. 2017a; Teng et al. 2017b). Stramenopiles also contain non-photosynthetic lineages, such as oomycetes, which behave as pathogens for many organisms, including humans (Dorrell et al. 2017).

The first evidence of P450 in marine macroalgae, including green algae, brown algae and red algae was obtained by CO-difference absorption spectra, though at rather low levels (Pflugmacher and Sandermann 1998). Active CYPs in marine macroalgae could remove marine xenobiotics by metabolizing several standard P450 substrates, which extended the green liver concept to these species (Pflugmacher and Sandermann 1998). The first identified stramenopile P450 sequence, CYP97E1, was cloned in the diatom Skeletonema costatum (Yang et al. 2003). Brown algae produce a vast array of specialized metabolites, such as diverse oxylipins (Kousaka et al. 2003; Ritter et al. 2008). The recently cloned CYP5164B1 in the brown alga Ectocarpus siliculosus converts 9 and 13-hydroperoxides of linoleic acid into oxylipin epoxyalcohols (Toporkova et al. 2017). Besides, blooming of CYPs in oomycetes has been reported with respect to their diversity and duplications, suggesting a role in adaptation of oomycetes to diverse ecological niches (Sello et al. 2015). However, stramenopile P450 diversity remains relatively unexplored, especially in the recently sequenced brown algae and diatoms. Taking into account the great importance of CYPs in metabolism and the paucity of knowledge about them in marine stramenopiles, this work performed systematic identification and comparison of $\mathrm{P} 450 \mathrm{~s}$ within representative lineages of stramenopiles to provide a detailed characterization and evolutionary history of the CYPome in this particular lineage. 


\section{Materials and methods}

\section{Data source}

The most up-to-date genomes of the three brown algae (Ectoparpus siliculosus, Cladosiphon okamuranus and Saccharina japonica), the three diatoms (Phaeodactylum tricornutum, Thalassiosira pseudonana and Fragilariopsis cylindrus) and the Nannochloropsis gaditana are available from public databases. Briefly, genome sequences of E. siliculosus V2016 version were downloaded from the we bsite http://bioinformatics.psb.ugent.be/orcae/overview/Ectsi (Cormier et al. 2016). Sequences of C. okamuranus were downloaded from http://marinegenomics.oist.jp/algae/ (Nishitsuji et al. 2016). Sequences of S. japonica were downloaded from NCBI https://www.ncbi.nlm.nih.gov/. Sequences of F. cylindrus were downloaded from JGI https://genome.jgi.doe.gov/portal (Grigoriev et al. 2014). Sequences of $P$. tricornutum, $T$. pseudonana, a $\mathrm{n} \mathrm{d} N$. gaditana were downloaded from ENSEMBL http://protists.ensembl.org/info/website/ftp/index.html (Kersey et al. 2018). Besides, the publicly available P450 sequences from two oomycetes Phytophthora sojae and Phytophthora ramorum, and other species (CYP51 and CYP97 from animal, plants, fungi, bacteria) were retrieved from the P450 homepage http://drnelson.uthsc.edu/CytochromeP450.html (Nelson 2009). Add ref here to transcriptomic datasets.

\section{Identification of P450s}

The P450 genes within stramenopiles were identified as described below. First, the P450 domain PF00067 was downloaded from the Pfam website http://pfam.xfam.org/ (Finn et al. 2016). HMMER3 http://hmmer.org/ (Eddy 2011) software with the default parameters was used to search for PF00067 domain in the proteomes of each species including the three brown algae, three diatoms and $N$. gaditana. Then, we used acquired proteins as query sequences for tBlastn and Blastp searches (Lobo 2012) against the genome sequences and predicted proteome sequences, respectively. Redundant sequences were discarded from the data set to obtain unique P450 genes. All of the obtained P450s were further manually examined using the NCBI online BLAST tool https://blast.ncbi.nlm.nih.gov/Blast.cgi to confirm the genes' annotation. The online InterProScan program http://www.ebi.ac.uk/interpro/search/sequence-search was used to confirm the presence of P450 domain in each sequence. All the putative P450s were named by David R. Nelson based on the existing nomenclature criteria, that is, $>40 \%$ sequence identity for assigning a family, $>55 \%$ for a subfamily and $<40 \%$ for a new family. Family assignment was further confirmed by phylogenetic reconstructions.

\section{Sequence structure analysis}

For each lineage (i.e. oomycetes, brown algae and diatoms), sequence structural features were explored to reveal a lineage-specific conservation pattern. Multiple protein sequence alignments were obtained using the hmmalign program in HMMER 3.1 package, with CYP protein sequences and PF00067.hmm as input files. Sequences logos of conserved motifs were generated using the online Weblogo program http://weblogo.threeplusone.com/create.cgi (Crooks et al. 2004) based on the sequence alignment results. Intron and exon information of the CYPs was obtained from their GFF3 files and was used for the graphic display using the Gene Structure Display Server of Peking University http://gsds.cbi.pku.edu.cn (Hu et al. 2014). Subcellular protein localization was predicted using ASAFind http://rocaplab.ocean.washington.edu/tools/asafind (Gruber et al. 
2015) and Euk-mPLoc 2.0 http://www.csbio.sjtu.edu.cn/bioinf/euk-multi-2/ (Chou and Shen 2010). The protein transmembrane helices were predicted by TMHMM server 2.0 http://www.cbs.dtu.dk/services/TMHMM/ . The distribution of P450s on chromosome or scaffold was displayed using Mapchart (Voorrips 2002). Genes separated by 0-5 genes were suggested to be tandem duplicates (Yu et al. 2015; Zhao et al. 2012).

\section{Phylogenetic analysis}

P450 protein sequences in stramenopiles were extracted and aligned using CLUSTAL Omega http://www.ebi.ac.uk/Tools/msa/clustalo/ (Sievers et al. 2011). The alignment file was used as input for MEGA 7.0 (Kumar et al. 2016). Maximum likelihood (ML) phylogenetic trees were constructed with the best substitution models Le-Gascuel (LG) + gamma (G) + Freqs. (F) model, as predicted by the menu 'Find best DNA/protein models' in MEGA 7.0. Bootstrap with 1000 replicates was performed to obtain the confidence support level. Besides, the phylogenetic relationships among the of CYP51 and CYP97 families, including stramenopiles, plants (red algae, green algae and higher plants), animals, bacteria and fungi, were reconstructed using protein sequences to investigate the phylogeny origin of these two global CYP families in stramenopiles.

\section{Evolutionary history analysis}

The ratio of non-synonymous $(\mathrm{d} N)$ to synonymous $(\mathrm{d} S)$ nucleotide substitution rates $(\mathrm{d} N / \mathrm{d} S=$ $\omega)$ provides information about the evolutionary forces operating on a gene. As positive selection promotes non-synonymous substitutions, an $\omega>1$ is considered indicative of positive selection, while genes under negative selection show an $\omega<1$. When genes are kept under neutral evolution, rates of synonymous and non-synonymous substitution are equal and $\omega$ $=1$ (Teng et al. 2017a). Thus, we estimated the selection pressure direction and strength of each CYP family by assessing $\omega$ values. The coding sequences of CYPs were translated into amino acid sequences using a local perl script. Alignment of these amino acid sequences was performed using CLUSTAL Omega http://www.ebi.ac.uk/Tools/msa/clustalo/ (Sievers et al. 2011). Codon alignments were generated using the protein sequence alignments as a guide (Suyama et al. 2006). The corresponding gene tree of each family was generated using MEGA 7.0. Then we used one ratio model $(\mathrm{M}=0)$ of Codeml program in the PAML package (Yang 2007) to calculate $\omega$ within each CYP family. Besides, site model, free ratio model, branch specific model and branch-site model were used to detect positively selected genes. To elucidate the evolutionary process of CYP families, we performed family history reconstruction using the COUNT software with default parameters (Csüös 2010). This software implements phylogenetic birth-and-death model, in which family size evolution on an edge is governed by a linear birth-and-death model. Ancestral reconstruction can be carried out by computing posterior probabilities for the family sizes at inner nodes. The species tree including the three brown algae and three diatoms, together with the numerical gene profiles (number of genes present in each family per species) were input into COUNT software and the data were analyzed using the Dollo parsimony model.

\section{Expression profile analysis}

Previous published microarray data from E. siliculosus were used to explore the expression levels of P450s in response to abiotic stresses, including hyposaline stress, hypersaline stress, oxidative stress, and $\mathrm{Cu}$ stress (Dittami et al. 2009; Ritter et al. 2014). The expression level was determined by averaging the expression values (previously quantile normalized by Roche 
NimbleGen, Madison, WI, USA) of four replicates for each experimental condition. Furthermore, expression levels of $S$. japonica P450s were examined in different life cycle stages including sporophytes, male gametophytes, and female gametophytes. Their transcriptomes were sequenced by our group using Illumina 2500, with three biological replicates in each life stage. The SRA accession numbers in the NCBI database are SRR5860560, SRR5860561, SRR5860562, SRR5860563, SRR5860564, SRR5860565, SRR5860566, SRR5860567, and SRR5860568. Gene expression levels were estimated by FPKM (Fragments Per Kilobase of transcript per Million fragments mapped). Differential expression analysis among life stages was performed using the DESeq (Love et al. 2014) R package using a model based on the negative binominal distribution. The resulting $\mathrm{P}$ values were adjusted using the Benjamini and Hochberg's approach for controlling the false discovery rate (Benjamini and Hochberg 1995). Genes with an adjusted $\mathrm{P}$ value $<0.05$ found by DESeq were assigned as differentially expressed. Transcriptome-wide microarray expression data of diatoms were acquired from the pan-transcriptomic analysis (Ashworth et al. 2016) http://networks.systemsbiology.net/diatom-portal/. The integrated data used in this analysis included: silica, iron, and nitrogen limitation, low temperature and elevated $\mathrm{pH}$ (Mock et al. 2008), exposure to pollutant benzo[a]pyrene (Carvalho et al. 2011), silica limitation (Sapriel et al. 2009), high light (Nymark et al. 2009), exposure to cadmium (Brembu et al. 2011), exposure to red, blue and green light (Valle et al. 2014). The microarray data were converted to $\log 2$ expression ratios $v s$. within-experiment control samples.

\section{Results and discussion}

\section{Identified CYPomes in Stramenopile species}

Genome-wide identification of P450s in three brown algae, three diatoms and $N$. gaditana revealed discrete gene numbers in each species of the three lineages (Fig. 1, Table S1, Fig. $\mathrm{S} 1)$. The P450 count in brown algae selected in this study ranged from 12 in E. siliculosus to 22 in C. okamuranus. The diatoms, on the other hand, showed the highest P450 number of 23 in F. cylindrus. Partial sequences, possibly pseudogenes, were also identified in these species, including one in P. tricornutum, two in $S$. japonica, three in $C$. okamuranus, seven in $F$. cylindrus, and one in $N$. gaditana. When naming partial sequences, we added a letter $\mathrm{P}$ after the normal nomenclature. Annotation of P450 families and subfamilies revealed variable distribution within the stramenopiles. In total, three brown algae and three diatoms have 25 families as defined in the standardized nomenclature for P450 sequences. Comparative analysis revealed that the three diatoms have higher P450 diversity than the three brown algae and the two oomycetes according to the number of families, whereas the two oomycetes showed the lowest diversity (Fig. 1). The three brown algae share six families, the three diatoms share three families, and all the brown algae, diatoms and $N$. gaditana share only two families, whereas they have none in common with the oomycetes $P$. ramorum and $P$. sojae. As oomycetes P450s have been already reported (Sello et al. 2015), we focused in this study on brown algae and diatoms.

CYP51 and CYP97 catalyze essential reactions conserved in the plant kingdom (Mizutani and Ohta 2010). Both of them were present in every alga examined (Fig. 1a). All of the CYP51 belong to subfamily CYP51C by definition. Notably, the brown alga $C$. okamuranus has an expanded CYP51 family, whereas other species contain only one copy of CYP51 in each genome. Another conserved family across species is CYP97. Most species contain two CYP97 members, with one belonging to CYP97E and the other CYP97F. F. cylindrus has one 
more CYP97F. P. tricornutum has two more CYP97E, with one CYP97E are partial sequence. Another shared family between brown algae, diatoms and $N$. gaditana is CYP5160. One CYP5160 exists in the three brown algae, P. tricornutum and $N$. gaditana whereas it is absent in T. pseudonana and F. cylindrus. CYP5161, CYP5163, CYP5164 and CYP5162 only occur in brown algae, of which the first three have several members per genome, whereas the later one CYP5162 features a single member. Among diatom-specific families, a single CYP5021 exists in all the three diatoms. CYP5022 and CYP5023 exist in T. pseudonana and $F$. cylindrus, while CYP5165 exists in P. tricornutum and F. cylindrus. In order to examine whether these families are indeed lineage-specific, we explored the transcriptomic database MMETSP (Keeling et al. 2014) and 1KP (https://db.cngb.org/onekp) to see the CYP distribution in related species. In close stramenopile relatives (Bolidomonas pacifica (?) close to diatoms and Vaucheria litorea (?) close to brown algae), identified CYP did not cluster with any lineage-specific CYPs (Fig. S2). Performing BLAST against 1KP database further supported that the brown algae-specific CYP families were only found in brown algae, CYP5023 was not found in the database, whereas diatoms' CYP5021, CYP5022 and CYP5165 were also found in other lineages, such as Haptophytes, suggesting they are not restricted to diatoms (Table S2). Notably, eight new families were found in F. cylindrus, that is, CYP5713, CYP5714, CYP5715, CYP5716, CYP5717, CYP5718, CYP5719, and CYP5720, suggesting their potential roles in adaptation to particular habitats. Indeed, $F$. cylindrus is a cold-adapted diatom, which lives in the extreme environment of the Southern Ocean, where photosynthesis is limited by large seasonal fluctuations in light, temperature and the extent of sea ice (Mock et al. 2017). We further found that these new families in $F$. cylindrus have diversified greatly. In detail, CYP5713A1 showed $27 \%$ identity to Danio rerio CYP7A and the top hits are animal CYP7. This family is pretty ancient, being conserved in animal and choanoflagellates and maybe up to fungi (Nelson et al. 2013). Having this in diatoms too may indicate that this is a much more ancient sequence, with lots of secondary losses (or technical biases like incomplete genome coverage). This is consistent with the basal position of this sequence grouping with CYP5164 and CYP51. CYP5714A1 showed 25\% identity to oomycete Saprolegnia parasitica. CYP5715A1 showed 20\% identity to CYP51C8 of $C$. okamuranus. CYP5716A1 showed 24\% identity to CYP5020A1 of $T$. pseudonana. CYP5717A1 showed 25\% identity to CYP5615A10 of $S$. parasitica. CYP5718A1 showed 25\% identity to CYP5658A1 of protist Acanthamoeba castellanii. CYP5718B1 showed 29\% identity to CYP5658A1 of A. castellanii. CYP5719A1 showed 22\% identity to CYP51C6 of C. okamuranus, which also indicates an ancient origin. CYP5720A1 showed $30 \%$ identity to CYP5710A1 of $N$. gaditana. The great P450 diversity of this ice diatom might contribute to its cold-adapted character. The maximum length of the CYP proteins occurred in F. cylindrus CYP5720A1 of 1273 amino acids (Table S3). P450s fused to redox partners are well documented in the literature (Sello et al. 2015; Guengerich and Munro 2013). The longer sequence of CYP5720A1 came from fusion to P450 reductase in the C-terminal of the protein. This kind of fusion also occurred in the CYP5710A1 of $N$. gaditana. Only twelve CYP protein sequences contained chloroplast-targeting peptides, six of which are CYP97 family members, consistent with the fact that CYP97 are involved in carotenoid metabolism in chloroplast, which plays essential roles in light-harvesting and photoprotection in plant kingdom (Mizutani and Ohta 2010; Tian et al. 2004). . On the other hand, most P450s were found to be endoplasmic reticulum located, which fits the canonical view of P450s bound to the cytoplasmic surface of the endoplasmic reticulum (Mizutani and Ohta 2010; Kelly et al. 2009). 


\section{Phylogenetic analysis}

To illustrate the relationship among these P450 families, a phylogenetic tree of stramenopile P450s was constructed using the full length of the protein sequences after removing the fused reductase parts. The results showed that some P450s names should be corrected by phylogenetic reconstruction. CYP5015G1, CYP5015G2 and CYP5015G3 of P. sojae should be the CYP5015G3, CYP5015G1 and CYP5015G2 orthologs, respectively. CYP5164C3 sequence was changed to CYP5164A4. The CYP5164B and C subfamilies were merged. After these corrections, the distribution on the tree agreed well with their family and subfamily assignment. Genes belonging to the same family grouped together robustly (Fig. 2). Phylogenetic analysis supported the high diversity of P450s in stramenopiles. According to the definition of a clan, where the reproducible clusters end and the variability begins in the deepest branches (Nelson 2017), we defined three clans in stramenopiles, including CYP51 clan shared with plants and animals, CYP97 clan shared with plants, and CYP5014 clan in oomycetes. CYP5715A1 and CYP5713A1 of F. cylindrus, CYP5166A1 of $P$. tricornutum and brown algae-specific CYP5164 grouped with the ancient CYP51 clan, reflecting their old origination or slow evolution compared with other families in brown algae or diatoms. CYP5692A2 of $N$. gaditana belonged to CYP5014 clan and showed a close relationship with CYP5017 of oomycetes. CYP5018A1 of T. pseudonana weakly grouped with CYP5014 clan. Besides, CYP51C of brown algae formed a larger branch than those of diatoms, resulting from the blooming of CYP51s from C. okamuranus. As the most conserved P450 family, CYP51 is widely distributed in all the biological kingdoms and is involved in the essential sterol biosynthesis pathway (Qi et al. 2006). 3 CYPs from brown algae and diatoms should likely come from a direct descendant of the oldest eukaryotic host. Both CYP51 and CYP97 showed a close relationship with red algae, green algae and higher plants. In most organisms that have been examined, only a single CYP51 gene exists, while several plants and fungi contain more than one copy, e.g. rice has ten, Arabidopsis thaliana has two, and some Aspergillus species have two (Nelson et al. 2004; Lepesheva and Waterman 2007). Most CYP51s across the different kingdoms were thought to have a single strictly conserved function in the synthesis of essential sterols, while some of those additional duplicates (e.g., CYP51H10 in oat) are demonstrated to be involved in the synthesis of antimicrobial compounds avenacins or triterpenes (Qi et al. 2006; Geisler et al. 2013). The identity between the two CYP51s from A. thaliana is $72 \%$. It was reported that one CYP51 from A. thaliana is an expressed pseudogene, while only CYP51G1 is functional (Kim et al. 2005). Despite the roles of the expanded CYP51s in C. okamuranus remain unknown, CYP51C1 of C. okamuranus is clearly orthologous to the other two CYP51C1 in S. japonica and E. siliculosus. This C. okamuranus ortholog is $87 \%$ identical to CYP51C1 of $S$. japonica, and less than $78 \%$ to the other seven $C$. okamuranus CYP51 sequences (Table S4), whereas the identities between the eight CYP51s from C. okamuranus range from 51\% between CYP51C3 and CYP51C8 to 89\% between CYP51C2 and CYP51C4. Furthermore, all the eight CYP51s have the four conserved enzyme functional motifs, suggesting that CYP51s in C. okamuranus are probably not pseudogenes but rather are evolving new functions, convergently to what happened in some terrestrial plants.

Despite highly variable sequences of these P450s, the sequence analysis showed some well conserved regions which correspond to the conserved enzyme functional motifs. There are four widely recognized consensus motifs AGXDTT, EXXR, PERF, and FXXGXXXCXG, which are critical for the detection of P450s in genomes (Kelly et al. 2009; Deng et al. 2007). Sequence logo analysis showed that these conserved motifs were present in oomycetes, diatoms and brown algae (Fig. S4). Gene structure analysis revealed that intron numbers vary dramatically between brown algae and diatoms (Fig. S5). The average intron number of 
brown algae P450s is seven, whereas that in diatoms is only one. Generally, the genes within the same family have almost the same intron number. In brown algal P450s, the intron number ranged from three in CYP97E8 of $S$. japonica and CYP97E9 of C. okamuranus to 13 in CYP5161D1 of $S$. japonica. A large part of brown algal P450 (34\%, 17 out of 49) have nine introns. Notably, the CYP51C1 of $S$. japonica and E. siliculosus, as well as the seven CYP51C of $C$. okamuranus contain nine introns, indicating the ancestor of brown algae CYP51C contained nine introns. On the contrary, in diatom P450s, the intron number ranged from 0 to 4 , with a large part of them $(44 \%, 19$ out of 43$)$ have no intron. Csuros et al. globally surveyed 100 complete genomes and inferred that evolution of eukaryotic genes was dominated by intron loss and the ancestor of 'SAR clade' is intron-rich (Csuros et al. 2011). The whole genome of $T$. pseudonana contain an average of 1.4 introns per gene. By evaluation of intron positions conservation, Roy and Penny found T. pseudonana has lost the majority of the numerous introns present in the 'SAR clade' ancestor (Roy and Penny 2007). These results revealed that intron loss occurred in diatoms P450s during the course of evolution.

\section{P450 evolutionary dynamics in brown algae and diatoms}

Based on naming and classification of families and subfamilies, further dynamics study was performed to assess the P450 evolutionary history within photosynthetic stramenopiles ochrophytes. Using the Dollo parsimony principle (Farris 1977; Almeida et al. 2016), combined with the results from 1KP transcriptome database, we estimated the P450 families that were gained or lost at each node of the algae tree(Fig. 3). In the common ancestor of brown algae and diatoms, there were six P450 families, including CYP51, CYP97, CYP5160, CYP5021, CYP5022, and CYP5165. Then, three families, that is, CYP5021, CYP5022, and CYP5165 were lost in brown algae. CYP5161, CYP5162, and CYP5164 were acquired in the ancestor of brown algae, followed by acquisition of CYP5163 in the ancestor of E. siliculosus and $C$. okamuranus. Alternatively, some of those CYPs may be highly diverging forms of otherwise conserved family. This is at least the case for CYP5164B1 that branches with CYP74 from eukaryotes and bacteria (Toporkova et al. 2017). Diatoms can produce a various set of epoxy alcohols, similar to those made by brown algae (Andreou et al. 2009). Thus, if they have no CYP74/CYP5164, this mean that either this enzyme diverged in terms of sequence until the point that it is not recognizable, or that allene oxide synthase activity was reacquired in diatoms from a different CYP paralog. The same is true for oomycetes, which are also able to produce epoxy alcohols from PUFAs (Andreou et al. 2009). The diatoms, on the other hand, seem to have undergone more frequent gain or loss events. CYP5023 was acquired in their ancestor. Subsequently, T. pseudonana gained four families, CYP5018, CYP5019, CYP5020 and CYP5024, and lost two families CYP5160 and CYP5165. Then, $P$. tricornutum gained CYP5166 and lost CYP5022 and CYP5023, whereas $F$. cylindrus lost CYP5160 and gained families CYP5712-5720. At last, diatom $F$. cylindrus contains the most CYP families of 15, followed by nine families in $T$. pseudonana, seven families in $E$. siliculosus and $C$. okamuranus, six families in $S$. japonica and $P$. tricornutum. Though drawing this evolutionary process, we cannot rule out other possibilities due to the biases linked to birth/death estimations in the context of multiple losses and heterogeneity in genome coverage. The dynamic changes of P450 families revealed that brown algae and diatoms evolved distinct P450 composition in their genomes, strongly suggesting their evolutionary adaptation to different coastal ecosystem.

6lyOn the other hand, tandem duplication rarely occurred in diatoms examined, with only CYP5165A1 and CYP5165A2 on chromosome 1 of P. tricornutum. 
Higher evolutionary rate may reflect the adaption to different substrates or emerging new functions. Lower evolutionary rate, on the other hand, would indicate the functional conservation or a strict substrate specificity (Parvez et al. 2016). To gain some insight into evolutionary rate and selection pressure on the evolution of the P450s, we compared $\omega$ values (ratio of non-synonymous to synonymous codon substitutions, $\mathrm{d} N / \mathrm{d} S$ ) in main families of brown algal and diatom P450. The one-ratio model of Codeml (one $\omega$ ratio for each family tree) showed that all the P450 families examined were have an $\omega$ of less than 0.4 (Fig. 4a). Under the site model that allows the $\omega$ ratio to vary among sites (M7 and M8), no positive selected site was detected (Table S5). CYP5163 sequences of brown algae had higher $\omega$ value than other families, maybe in relation with the fact it emerged recently, given that $\omega$ values decay over time, due to the accumulation of synonymous substitutions. CYP51 and CYP97 in diatoms have lower $\omega$ values than those of brown algae, indicating the slow evolutionary rates in diatoms. Considering that the higher $\omega$ ratio in brown algal CYP51 may result from the expansion of CYP51 in C. okamuranus, we further applied the free-ratio model (independent $\omega$ ratio for each branch) to test the selection along each branch (Fig. 4b). Interestingly, we detected higher $\omega$ value along the branches leading to the $C$. okamuranus CYP51C2-C8 compared with other CYP51C1 genes. To further confirm this result, branch specific model $(\mathrm{M}=2)$ and branch-site model $(\mathrm{M}=2$, Nssites $=2)$ were used to check the selection pressure of the expanded CYP51Cs in C. okamuranus. Consistently, the CYP51C2C8 clade exhibited higher $\omega$ value $(\omega=0.5180)$ than other clades, though no site under positive selection was detected (Table S5). This result suggested that the relaxed selection pressure might contribute to the remaining of multiple CYP51C in C. okamuranus and further to acquisition of new activity or a change of function emerging post-duplication. On the contrary, the CYP51s of diatoms and $N$. gaditana were found under strong negative selection, indicating a functional conservation in the course of evolution.

\section{Transcriptome analysis and evolution of two critical steps in the oxylipin synthesis pathway among stramenopiles}

We used publicly available microarray datasets from E. siliculosus (Dittami et al. 2009; Ritter et al. 2014), diatoms (Ashworth et al. 2016) and our RNA-seq datasets of the three life stages o f $S$. japonica including sporophytes, male gametophytes and female gametophytes to investigate the expression profiles of $P 450$ genes. Among the fifteen P450s of S. japonica, four genes were not expressed in all the three life stages, two of which are truncated pseudogenes (Fig. 5a). The other two (CYP5161C3 and CYP5164B3) may have become pseudogenes after duplication or evolved new function not identified in our study, such as expression induced by herbivore grazing, as it may occur with other kelps (Ritter et al. 2017). Seven genes showed significantly higher expression levels in sporophytes (adjusted $p$ value $<$ 0.05), including CYP5164B2, CYP5161C1, CYP5161C2, CYP5164C1, CYP51C1, CYP5161D1 and CYP5160B2. In previous study, Toporkova et al. (2017) cloned CYP5164B1 of $E$. siliculosus and showed it worked as epoxyalcohol synthase, converting fatty acid hydroperoxides into oxylipin epoxyalcohols. Brown algae possess a great diversity of oxylipins (Ritter et al. 2008; Proteau and Gerwick 1992). Considering that CYP5164s expanded in $S$. japonica and is mainly expressed in sporophytes, they may contribute to high amounts of oxylipins biosynthesis in this large kelp. Besides, only one gene, CYP97F8 was highly expressed in male and female gametophytes, suggesting its pivotal roles in gametophyte development.

Transcriptome analysis of $E$. siliculosus showed that most $P 450$ genes are responsive to at 
least one stress condition (fold change $>2$ compared with control) (Fig. 5b). Ritter et al. demonstrated that copper treatment $(\mathrm{Cu})$ could induce oxidative stress and activate oxylipin biosynthesis in E. siliculosus and kelp Laminaria digitata (Ritter et al. 2008; Ritter et al. 2014). Two genes are responsive to $\mathrm{Cu}$ stress, with one gene CYP5164A2 up-regulated while the other gene CYP97F4 down-regulated, suggesting that CYP5164A2 either participates in oxylipin synthesis, or in detoxification of environmental chemicals. Moreover, CYP5164A2 was also up-regulated under oxidative and hyposaline stresses, which further supports the anti-oxidation roles of this gene. Besides, CYP5164B1 of E. siliculosus, which has been proved to synthesize oxylipin epoxyalcohol (Toporkova et al. 2017) was up-regulated under hypersaline stress while down-regulated under oxidative stress, indicating it is involved in stress response. Additionally, CYP97F4, which participates in photosynthetic pigment synthesis, was down-regulated under all conditions, indicating that photosynthesis was vulnerable under these stress conditions.

Diatoms transcriptomic analysis revealed some CYPs are responsive to specific conditions. Similar to E. siliculosus, CYP97 in diatoms were also down regulated across many conditions, such as silicon, iron and nitrogen limitation in T. pseudonana, short term high light and blue light treatment in $P$. tricornutum (Fig. 5c,d), probably reflecting a negative-feedback regulation of photosynthesis. Besides, in T. pseudonana, CYP5024A1 and CYP5018A1 were up regulated under low temperature and down regulated under silicon limitation, CYP5019A1 was up regulated when exposed to the pollutant benzo[a]pyrene at a sub-lethal concentration. On the other hand, in $P$. tricornutum, strong and rapid response to the changes in irradiance was observed in CYP5166A1 and CYP5165A1. CYP5166A1 was up regulated after transfer to high light conditions, indicating its potential roles in protecting the organism from photooxidative damage to the photosynthetic machinery. CYP5165Al was up regulated under red, green and blue light at the initial response phase, followed by the intermediate and late acclimation phase, suggesting its critical roles in sensing spectral quality.

Our new transcriptomic data on P450 genes, as well as our previous studies on the LOX gene family (Teng et al., 2017b) can now be integrated with metabolic data to propose a first comparative model of the first two steps in the oxylipin synthesis pathway among stramenopiles (Fig. 6). Lineage-specific models have already been published previously, at a time when few whole-genome data were available for stramenopiles (Andreou et al., 2009). Now both genomic and metabolomic data are available for Ectocarpus siliculosus, and genome and metabolome data are available for two species pairs from the same genus in two other stramenopiles. Genome data from $S$. japonica can be compared to metabolome data from S. angustata (Boonprab et al. 2003b; Boonprab et al. 2003a), whereas genome data from T. pseudonana can be related with metabolic data from T. rotula (Barofsky and Pohnert 2007; D'Ippolito et al. 2006). It is not yet possible to assign all P450 paralogs to a specific enzymatic activity, but our data already provide some clarifications. First, absence of a CYP74/CYP5164-like gene in Thalassosiria correlates with absence of an hydroperoxide lyase (HPL) activity linked to a P450. On the contrary, CYP5713A1 from another diatom, Fragillariopsis cylindricus, would be an interesting candidate to test if a P450-linked HPL activity is still retained in some diatoms. Second, it is already clear that land plants and brown algae independently underwent diversification of either CYP74 or CYP5164. From that we can expect that the biochemical activities are differently subfunctionalized across the angiosperm and stramenopile lineages. 


\section{Conclusions}

In this study, we systematically investigated the P450s diversity and evolution within stramenopiles species, which evolved from secondary endosymbiosis events. Three clans were defined in this lineage, including CYP51 clan, CYP97 clan and CYP5014 clan. CYPomes characteristics differ greatly among oomycetes, brown algae and diatoms. Phylogenetic analysis revealed that P450s of diatoms and brown algae showed a closer relationship than with oomycetes P450s. The CYP51 family expanded in brown alga $C$. okamuranus. 1KP transcriptome analysis revealed that some families, such as CYP5021 and CYP5022 are not restricted to diatoms. Gene gain and loss patterns revealed that six CYP families - CYP51, CYP97 and CYP5160, CYP5021, CYP5022 and CYP5165 predated the split of brown algae and diatoms. After they diverged, diatoms gained more CYP families, especially for the cold-adapted species $F$. cylindrus, in which eight new CYP families were found. Expression analysis showed that most P450s in S. japonica were highly expressed in sporophytes, which suggests a potential role in large kelp development. E. siliculosus and diatom P450s, on the other hand, were found responsive to many stressful or irradiance conditions, suggesting their important roles in response to environmental stimulus. The information provided in this study will be helpful in further investigating P450 roles in this diversified lineage.

\section{Acknowledgements}

This work was supported by Central Public-interest Scientific Institution Basal Research Fund CAFS (2017HY-YJ01); National key Research and Development Plan (2016YFC1402102-2); National Natural Science Foundation of China (41676145); Shandong key Research and Development Plan; Qingdao Municipal Science and Technology plan project (17-1-1-96-jch); Special Scientific Research Funds for Central Non-Profit Institutes, Yellow Sea Fisheries Research Institute, Chinese Academy of Fishery Sciences (20603022016010, 20603022016001); China Agriculture Research System (CARS-50); Taishan Scholars Funding; AoShan Talents Program (No. 2015ASTPES03); the Science Fund for Distinguished Young Scholars of Shandong Province (JQ201509); and Projects of International Exchange and Cooperation in Agriculture, Ministry of Agriculture and Rural Affairs of China-Science, Technology and Innovation Cooperation in Aquaculture with Tropical Countries along the Belt and Road. G.V.M. benefited from the support of the French Government via the National Research Agency investment expenditure program IDEALG (ANR-10-BTBR-04) and from Région Bretagne via the grant «SAD2016-METALG (9673) ».

\section{Reference}

Aleoshin VV, Mylnikov AP, Mirzaeva GS, Mikhailov KV, Karpov SA (2016) Heterokont PredatorDevelorapax marinusgen. et sp. nov. - A Model of the Ochrophyte Ancestor. Frontiers in Microbiology 7 (16)

Almeida D, Maldonado E, Khan I, Silva L, Gilbert MTP, Zhang G, Jarvis ED, O'Brien SJ, Johnson WE, Antunes A (2016) Whole-Genome Identification, Phylogeny, and 
Evolution of the Cytochrome P450 Family 2 (CYP2) Subfamilies in Birds. Genome Biology \& Evolution 8 (4):1115-1131

Andreou A, Brodhun F, Feussner I (2009) Biosynthesis of oxylipins in non-mammals. Progress in Lipid Research 48 (3-4):148-170

Ashworth J, Turkarslan S, Harris M, Orellana MV, Baliga NS (2016) Pan-transcriptomic analysis identifies coordinated and orthologous functional modules in the diatoms Thalassiosira pseudonana and Phaeodactylum tricornutum. Marine Genomics 26:21

Barofsky A, Pohnert G (2007) Biosynthesis of polyunsaturated short chain aldehydes in the diatom Thalassiosira rotula. Organic Letters 9 (6):1017-1020

Benjamini Y, Hochberg Y (1995) Controlling The False Discovery Rate - A Practical And Powerful Approach To Multiple Testing. Journal of the Royal Statistical Society 57 (1):289-300

Boonprab K, Matsui K, Akakabe Y, Yotsukura N, Kajiwara T (2003a) Hydroperoxyarachidonic acid mediated n-hexanal and (Z)-3- and (E)-2-nonenal formation in Laminaria angustata. Phytochemistry 63 (6):669

Boonprab K, Matsui K, Yoshida M, Akakabe Y, Chirapart A, Kajiwara T (2003b) C6aldehyde formation by fatty acid hydroperoxide lyase in the brown alga Laminaria angustata. Zeitschrift Für Naturforschung C 58 (4):207-214

Brembu T, Jørstad M, Winge P, Valle KC, Bones AM (2011) Genome-Wide Profiling of Responses to Cadmium in the Diatom Phaeodactylum tricornutum. Environmental Science \& Technology 45 (18):7640-7647

Carvalho RN, Bopp SK, Lettieri T (2011) Transcriptomics Responses in Marine Diatom Thalassiosira pseudonana Exposed to the Polycyclic Aromatic Hydrocarbon Benzo[a]pyrene. Plos One 6 (11):: e26985.

Chou K-C, Shen H-B (2010) A new method for predicting the subcellular localization of eukaryotic proteins with both single and multiple sites: Euk-mPLoc 2.0. PLoS One 5 (4):e9931

Cock JM, Sterck L, Ahmed S, Allen A, Amoutzias G, Anthouard V, Artiguenave F, Arun A, Aury JM (2012) The Ectocarpus genome and brown algal genomics. Elsevier Academic Press, San Diego, CA

Cock JM, Sterck L, Rouzé P, Scornet D, Allen AE, Amoutzias G, Anthouard V, Artiguenave F, Aury JM, Badger JH (2010) The Ectocarpus genome and the independent evolution of multicellularity in brown algae. Nature 465:617-621

Cormier A, Avia K, Sterck L, Derrien T, Wucher V, Andres G, Monsoor M, Godfroy O, Lipinska A, Perrineau M-M, Peer YVD, Hitte C, Corre E, Coelho SM, Cock JM (2016) Re-annotation, improved large-scale assembly and establishment of a catalogue of noncoding loci for the genome of the model brown alga Ectocarpus. New Phytologist 214 (1)

Crooks GE, Hon G, Chandonia JM, Brenner SE (2004) WebLogo: A Sequence Logo Generator. Genome Research 14 (6):1188-1190

Csüös M (2010) Count: evolutionary analysis of phylogenetic profiles with parsimony and likelihood. Bioinformatics 26 (15):1910-1912

Csuros M, Rogozin IB, Koonin EV (2011) A detailed history of intron-rich eukaryotic ancestors inferred from a global survey of 100 complete genomes. PLoS computational biology 7 (9):e1002150

D'Ippolito G, Cutignano A, Tucci S, Romano G, Cimino G, Fontana A (2006) Biosynthetic intermediates and stereochemical aspects of aldehyde biosynthesis in the marine diatom Thalassiosira rotula. Phytochemistry 67 (3):314-322

Debeljak N, Horvat S, Vouk K, Lee M, Rozman D (2000) Characterization of the mouse lanosterol 14a-demethylase (CYP51), a new member of the evolutionarily most 
conserved cytochrome P450 family. Archives of biochemistry and biophysics 379 (1):37-45

Deng J, Carbone I, Dean RA (2007) The evolutionary history of Cytochrome P450 genes in four filamentous Ascomycetes. Bmc Evolutionary Biology 7 (1):30

Dittami SM, Scornet D, Petit J-L, Ségurens B, Da Silva C, Corre E, Dondrup M, Glatting KH, König R, Sterck L (2009) Global expression analysis of the brown alga Ectocarpus siliculosus (Phaeophyceae) reveals large-scale reprogramming of the transcriptome in response to abiotic stress. Genome biology 10 (6):R66

Dorrell RG, Gile G, Mccallum G, Méheust R, Bapteste EP, Klinger CM, Brillet-Guéguen L, Freeman KD, Richter DJ, Bowler C (2017) Chimeric origins of ochrophytes and haptophytes revealed through an ancient plastid proteome. eLife 6:e23717

Eddy SR (2011) Accelerated Profile HMM Searches. Plos Computational Biology 7 (10):e1002195

Farris JS (1977) Phylogenetic Analysis Under Dollo's Law. Systematic Zoology 26 (1):77-88

Finn RD, Coggill P, Eberhardt RY, Eddy SR, Mistry J, Mitchell AL, Potter SC, Punta M, Qureshi M, Sangrador-Vegas A (2016) The Pfam protein families database: towards a more sustainable future. Nucleic acids research 44 (D1):D279-D285

Frommolt R, Werner S, Paulsen H, Goss R, Wilhelm C, Zauner S, Maier UG, Grossman AR, Bhattacharya D, Lohr M (2008) Ancient recruitment by chromists of green algal genes encoding enzymes for carotenoid biosynthesis. Molecular Biology \& Evolution 25 (12):2653-2667(2615)

Geisler K, Hughes RK, Sainsbury F, Lomonossoff GP, Rejzek M, Fairhurst S, Olsen CE, Motawia MS, Melton RE, Hemmings AM (2013) Biochemical analysis of a multifunctional cytochrome P450 (CYP51) enzyme required for synthesis of antimicrobial triterpenes in plants. Proceedings of the National Academy of Sciences of the United States of America 110 (35):E3360

Goldstone HM, Stegeman JJ (2006) A revised evolutionary history of the CYP1A subfamily: gene duplication, gene conversion, and positive selection. Journal of Molecular Evolution 62 (6):708-717

Grigoriev IV, Nordberg H, Shabalov I, Aerts A, Cantor M, Goodstein D, Kuo A, Minovitsky S, Nikitin R, Ohm RA (2014) The Genome Portal of the Department of Energy Joint Genome Institute. Nucleic Acids Research 42 (Database issue):26-31

Gruber A, Rocap G, Kroth PG, Armbrust E, Mock T (2015) Plastid proteome prediction for diatoms and other algae with secondary plastids of the red lineage. The Plant Journal $81(3): 519-528$

Guengerich FP, Munro AW (2013) Unusual cytochrome p450 enzymes and reactions. Journal of Biological Chemistry 288 (24):17065-17073

Hu B, Jin J, Guo A-Y, Zhang H, Luo J, Gao G (2014) GSDS 2.0: an upgraded gene feature visualization server. Bioinformatics 31 (8):1296-1297

Keeling PJ (2013) The Number, Speed, and Impact of Plastid Endosymbioses in Eukaryotic Evolution. Annual Review of Plant Biology 64 (1):583-607

Keeling PJ, Burki F, Wilcox HM, Allam B, Allen EE, Amaral-Zettler LA, Armbrust EV, Archibald JM, Bharti AK, Bell CJ (2014) The Marine Microbial Eukaryote Transcriptome Sequencing Project (MMETSP): Illuminating the Functional Diversity of Eukaryotic Life in the Oceans through Transcriptome Sequencing. Plos Biology 12 (6):429-440

Kelly DE, Kraševec N, Mullins J, Nelson DR (2009) The CYPome (cytochrome P450 complement) of Aspergillus nidulans. Fungal genetics and biology 46 (1):S53-S61

Kersey PJ, Allen JE, Allot A, Barba M, Boddu S, Bolt BJ, Carvalho-Silva D, Christensen M, Davis P, Grabmueller C (2018) Ensembl Genomes 2018: an integrated omics 
infrastructure for non-vertebrate species. Nucleic Acids Research 46 (Database issue):D802-D808

Kim HB, Schaller H, Goh C-H, Kwon M, Choe S, An CS, Durst F, Feldmann KA, Feyereisen R (2005) Arabidopsis cyp51 mutant shows postembryonic seedling lethality associated with lack of membrane integrity. Plant physiology 138 (4):2033-2047

Kousaka K, Ogi N, Akazawa Y, Fujieda M, Yamamoto Y, Takada Y, Kimura J (2003) Novel oxylipin metabolites from the brown alga Eisenia bicyclis. Journal of natural products $66(10): 1318-1323$

Kumar S, Stecher G, Tamura K (2016) MEGA7: Molecular Evolutionary Genetics Analysis version 7.0 for bigger datasets. Molecular biology and evolution 33 (7):1870-1874

Lepesheva GI, Waterman MR (2007) Sterol 14 $\alpha$-demethylase cytochrome P450 (CYP51), a P450 in all biological kingdoms. Biochimica et Biophysica Acta (BBA)-General Subjects 1770 (3):467-477

Li Z, Defoort J, Tasdighian S, Maere S, Van de Peer Y, De Smet R (2016) Gene Duplicability of Core Genes Is Highly Consistent across All Angiosperms. Plant Cell 28 (2):326344. doi:10.1105/tpc.15.00877

Liu Z, Tavares R, Forsythe ES, André F, Lugan R, Jonasson G, Boutet-Mercey S, Tohge T, Beilstein MA, Werck-Reichhart D, Renault H (2016) Evolutionary interplay between sister cytochrome P450 genes shapes plasticity in plant metabolism. Nature Communications 7:13026

Lobo I (2012) Basic Local Alignment Search Tool (BLAST). Journal of Molecular Biology 215 (3):403-410

Love MI, Huber W, Anders S (2014) Moderated estimation of fold change and dispersion for RNA-seq data with DESeq2. Genome Biology 15 (12):550

Mizutani M, Ohta D (2010) Diversification of P450 Genes During Land Plant Evolution. Annual Review of Plant Biology 61 (1):291

Mock T, Otillar RP, Strauss J, McMullan M, Paajanen P, Schmutz J, Salamov A, Sanges R, Toseland A, Ward BJ (2017) Evolutionary genomics of the cold-adapted diatom Fragilariopsis cylindrus. Nature:1-5

Mock T, Samanta MP, Iverson V, Berthiaume C, Robison M, Holtermann K, Durkin C, Bondurant SS, Richmond K, Rodesch M (2008) Whole-Genome Expression Profiling of the Marine Diatom Thalassiosira pseudonana Identifies Genes Involved in Silicon Bioprocesses. Proceedings of the National Academy of Sciences of the United States of America 105 (5):1579-1584

Moustafa A, Beszteri B, Maier UG, Bowler C, Valentin K, Bhattacharya D (2009) Genomic Footprints of a Cryptic Plastid Endosymbiosis in Diatoms. Science 324. doi:10.1126/science.1172983

Nelson DR (1998) Cytochrome P450 nomenclature. Methods in Molecular Biology 107:15

Nelson DR (2009) The Cytochrome P450 Homepage. Human Genomics 4 (1):59

Nelson DR (2017) Cytochrome P450 diversity in the tree of life. Biochimica Et Biophysica Acta

Nelson DR, Goldstone JV, Stegeman JJ (2013) The cytochrome P450 genesis locus: the origin and evolution of animal cytochrome P450s. Philosophical Transactions of the Royal Society of London 368 (1612):20120474

Nelson DR, Schuler MA, Paquette SM, Werck-Reichhart D, Bak S (2004) Comparative genomics of rice and Arabidopsis. Analysis of 727 cytochrome P450 genes and pseudogenes from a monocot and a dicot. Plant Physiology 135 (2):756-772

Nishitsuji K, Arimoto A, Iwai K, Sudo Y, Hisata K, Fujie M, Arakaki N, Kushiro T, Konishi T, Shinzato C (2016) A draft genome of the brown alga, Cladosiphon okamuranus, Sstrain: a platform for future studies of 'mozuku'biology. Dna Research 23 (6):561-570 
Nymark M, Valle KC, Brembu T, Hancke K, Winge P, Andresen K, Johnsen G, Bones AM (2009) An Integrated Analysis of Molecular Acclimation to High Light in the Marine Diatom Phaeodactylum tricornutum. Plos One 4 (11):e7743

Omura T, Gotoh O (2017) Evolutionary Origin of Mitochondrial Cytochrome P450. Journal of Biochemistry 161 (5):399-407

Parvez M, Qhanya LB, Mthakathi NT, Kgosiemang IK, Bamal HD, Pagadala NS, Xie T, Yang H, Chen H, Theron CW (2016) Molecular evolutionary dynamics of cytochrome P450 monooxygenases across kingdoms: Special focus on mycobacterial P450s. Scientific Reports 6 (33099):33099

Pflugmacher S, Sandermann H (1998) Cytochrome P450 monooxygenases for fatty acids and xenobiotics in marine macroalgae. Plant physiology 117 (1):123-128

Phillips IR, Shephard EA (1998) Cytochrome P450 protocols. Humana Press, New Jersey

Proteau PJ, Gerwick WH (1992) Cymathere ethers A and B: bicyclic oxylipins from the marine brown alga Cymathere triplicata. Tetrahedron letters 33 (31):4393-4396

Qi X, Bakht S, Qin B, Leggett M, Hemmings A, Mellon F, Eagles J, Werck-Reichhart D, Schaller H, Lesot A (2006) A different function for a member of an ancient and highly conserved cytochrome P450 family: From essential sterols to plant defense. Proceedings of the National Academy of Sciences of the United States of America 103 (49):18848-18853

Renault H, Bassard JE, Hamberger B, Werck-Reichhart D (2014) Cytochrome P450-mediated metabolic engineering: current progress and future challenges. Current Opinion in Plant Biology 19 (19C):27-34

Renault H, De MM, Jonasson G, Lara P, Nelson DR, Nilsson I, André F, Von HG, WerckReichhart D (2017) Gene duplication leads to altered membrane topology of a cytochrome P450 enzyme in seed plants. Molecular Biology \& Evolution

Ritter A, Cabioch L, Brilletguéguen L, Corre E, Cosse A, Dartevelle L, Duruflé H, Fasshauer C, Goulitquer S, Thomas F (2017) Herbivore-induced chemical and molecular responses of the kelps Laminaria digitata and Lessonia spicata. Plos One 12 (3): 0173315

Ritter A, Dittami SM, Goulitquer S, Correa JA, Boyen C, Potin P, Tonon T (2014) Transcriptomic and metabolomic analysis of copper stress acclimation in Ectocarpus siliculosus highlights signaling and tolerance mechanisms in brown algae. BMC plant biology 14 (1):116

Ritter A, Goulitquer S, Salaün JP, Tonon T, Correa JA, Potin P (2008) Copper stress induces biosynthesis of octadecanoid and eicosanoid oxygenated derivatives in the brown algal kelp Laminaria digitata. New Phytologist 180 (4):809-821

Roy SW, Penny D (2007) A very high fraction of unique intron positions in the intron-rich diatom Thalassiosira pseudonana indicates widespread intron gain. Molecular biology and evolution 24 (7):1447-1457

Sapriel G, Quinet M, Heijde M, Jourdren L, Tanty V, Luo G, Le Crom S, Lopez P (2009) Genome-wide transcriptome analyses of silicon metabolism in Phaeodactylum tricornutum reveal the multilevel regulation of silicic acid transporters. PLoS ONE 4 (10): 7458

Sello MM, Jafta N, Nelson DR, Chen W, Yu JH, Parvez M, Kgosiemang IKR, Monyaki R, Raselemane SC, Qhanya LB (2015) Diversity and evolution of cytochrome P450 monooxygenases in Oomycetes. Scientific Reports 5: 11572

Ševčíková T, Horák A, Klimeš V, Zbránková V, Demir-Hilton E, Sudek S, Jenkins J, Schmutz J, Přibyl P, Fousek J, Vl

ek Č, Lang BF, Oborník M, Worden AZ, Eliá š M (2015) Updating algal evolutionary relationships through plastid genome sequencing: did alveolate plastids emerge 
through endosymbiosis of an ochrophyte? Scientific Reports 5:10134. doi:10.1038/srep10134

Sievers F, Wilm A, Dineen D, Gibson TJ, Karplus K, Li W, Lopez R, McWilliam H, Remmert M, Söding J (2011) Fast, scalable generation of high- quality protein multiple sequence alignments using Clustal Omega. Molecular systems biology 7 (1):539

Suyama M, Torrents D, Bork P (2006) PAL2NAL: robust conversion of protein sequence alignments into the corresponding codon alignments. Nucleic acids research 34 (suppl_2):W609-W612

Syed K, Nelson DR, Riley R, Yadav JS (2013) Genomewide annotation and comparative genomics of cytochrome P450 monooxygenases (P450s) in the polypore species Bjerkandera adusta, Ganoderma sp. and Phlebia brevispora. Mycologia 105 (6):14451455. doi:10.3852/13-002

Teng L, Fan X, Xu D, Zhang X, Mock T, Ye N (2017a) Identification of Genes under Positive Selection Reveals Differences in Evolutionary Adaptation between Brown-Algal Species. Frontiers in Plant Science 8:1429

Teng L, Han W, Fan X, Xu D, Zhang X, Dittami SM, Ye N (2017b) Evolution and Expansion of the Prokaryote-Like Lipoxygenase Family in the Brown Alga Saccharina japonica. Frontiers in Plant Science 8:2018

Tian L, Musetti V, Kim J, Magallaneslundback M, Dellapenna D (2004) The Arabidopsis LUT1 locus encodes a member of the cytochrome P450 family that is required for carotenoid $\varepsilon$-ring hydroxylation activity. Proceedings of the National Academy of Sciences of the United States of America 101 (1):402

Toporkova YY, Fatykhova VS, Gogolev YV, Khairutdinov BI, Mukhtarova LS, Grechkin AN (2017) Epoxyalcohol synthase of Ectocarpus siliculosus. First CYP74-related enzyme of oxylipin biosynthesis in brown algae. Biochimica et Biophysica Acta (BBA) Molecular and Cell Biology of Lipids 1862 (2):167-175

Valle KC, Nymark M, Aamot I, Hancke K, Winge P, Andresen K, Johnsen G, Brembu T, Bones AM (2014) System Responses to Equal Doses of Photosynthetically Usable Radiation of Blue, Green, and Red Light in the Marine Diatom Phaeodactylum tricornutum. Plos One 9 (12):e114211

Voorrips RE (2002) MapChart: Software for the Graphical Presentation of Linkage Maps and QTLs. Journal of Heredity 93 (1):77-78

Yang S, Wu RS, Mok HO, Zhang Z, Kong RY (2003) Identification of a novel cytochrome P450 cDNA, CYP97E1, from the marine diatom Skeletonema costatum Bacillariophyceae. Journal of Phycology 39 (3):555-560

Yang Z (2007) PAML 4: Phylogenetic Analysis by Maximum Likelihood. Molecular Biology and Evolution 24 (8):1586-1591

Ye N, Zhang X, Miao M, Fan X, Zheng Y, Xu D, Wang J, Zhou L, Wang D, Gao Y (2015) Saccharina genomes provide novel insight into kelp biology. Nature communications 6:6986

Yu L, Tang W, He W, Ma X, Vasseur L, Baxter SW, Yang G, Huang S, Song F, You M (2015) Characterization and expression of the cytochrome P450 gene family in diamondback moth, Plutella xylostella (L.). Scientific Reports 5:8952

Zhao H, Ma H, Yu L, Wang X, Zhao J (2012) Genome-Wide Survey and Expression Analysis of Amino Acid Transporter Gene Family in Rice ( Oryza sativa L.). Plos One 7 (11):e49210 


\section{Figure legend}

Fig. 1 Statistics of P450 families in each stramenopile species. a CYP family member distribution. b Comparative analysis of the number of P450s and P450 families. C. P450 diversity percentage. It is represented by the percentage of the number of $\mathrm{P} 450$ families in the total number of P450s in an organism.

Fig. 2 Phylogenetic tree of P450s in stramenopile species. The ML tree was constructed using MEGA 7.0 with LG+G+F model. The branch names represent the P450 name, followed by the species names. The branches are colored to show their different taxonomic groups of oomycetes, brown algae, diatoms and $N$. gaditana, respectively. The number on the tree node means bootstrap (1000 replicates) values above $50 \%$.

Fig. 3 Evolutionary process of brown algae/diatoms CYP families. Green circles indicate the gain of the corresponding CYP families while red circles indicate their loss. Numbers along the phylogenetic tree denote the number of CYP families present in each moment of the alga evolution

Fig. $4 \omega$ values computed with Codeml branch model. a The $\omega$ values of CYP families using branch one ratio model $(\mathrm{M}=0)$. b The $\omega$ values in the CYP51 family. Blue number is the $\omega$ values of each gene under free ratio model $(M=1)$, red number is the $\omega$ values of different phylogenetic groups under specific branch model $(\mathrm{M}=2)$.

Fig. 5 Expression profiles of P450 genes. a Log10-transformed FPKM values in S. japonica. Black stars indicate sex-biased or sporophyte-biased expression (fold changes $>2$, adjusted $p$ value < 0.05). SP: sporophytes; FG: female gametophytes; MG: male gametophytes; b Log2transformed fold changes of expression levels compared to the control in E. siliculosus. hyper: hypersaline; hypo: hyposaline; oxi: oxidative; $\mathrm{Cu}_{-} 4$ : copper treated for $4 \mathrm{~h} ; \mathrm{Cu} 8$ : copper treated for $8 \mathrm{~h}$. Black stars indicate the $\log 2$ values of fold changes (treatments/control) $>1.0$ or $<-1.0$; $\mathbf{c} \log 2$-transformed fold changes of expression levels compared to the control in T. pseudonana. T, $\mathrm{Si}, \mathrm{Fe}, \mathrm{N}$ : low temperature, silicon, iron and nitrogen limitation; $\mathrm{BaP}$ : benzo[a]pyrene; $\mathrm{pH}$ : alkaline $\mathrm{pH}$; Black stars indicate the log2 values of fold changes (treatments/control) $>1.0$ or $<-1.0 ; \mathbf{d}$ Log2-transformed fold changes of expression levels compared to the control in P. tricornutum. Black stars indicate the $\log 2$ values of fold changes (treatments/control) $>1.0$ or $<-1.0$. 'NA' means the data is not available.

Fig. 6 Comparison of the two steps in the oxilipin synthesis pathway across stramenopiles.

Each of the color frame represents a synthetic pathway in one lineage. This is a single-species lineage for A. thaliana and E. siliculosus, whereas genomic and metabolomic data are pooled for two species from the same genus for Saccharina and Thalassiosiria. The peroxidation step in orange is always carried out by a LOX enzyme, wherease the hydroperoxilyase activity (in purple) can be carried out either by a P450 (Arabidopsis and the two brown algae) or by an enzyme from a different family in Thalassiosiria (blue frame).

\section{Supplementary material}

Fig. S1 Statistics of P450 families in each stramenopiles species.

Fig. S2 Phylogenetic tree of CYPs in brown algae, diatoms, Bolidomonas and Vaucheria. The ML tree was constructed using MEGA 7.0 with WAG+G model. The dark yellow branches are CYPs only 
occurred in brown algae. The blue branches are CYPs only occurred in diatoms. The red and green branched correspond to CYPs from Bolidomonas and Vaucheria, respectively.

Fig. S3 Phylogeny of brown algae and diatoms CYP51 and CYP97 with their counterparts in animal, plant, bacterial and fungi. The phylogenetic tree was constructed with MEGA 7 and displayed with Figtree, using the alignments of CYPs generated by hmmalign in HMMER program with CYP sequences and PF00067.hmm as input files. 16 animal CYP51s, 10 bacteria CYP51s, 27 plant CYP51s, 21 fungal CYP51s and 16 plant CYP97s were extracted from the CYP homepage http://drnelson.uthsc.edu/CytochromeP450.html.

Fig. S4 Sequence logos of the conserved CYP motifs from the three lineages of oomycetes, diatoms and brown algae. Multiple alignments of CYP proteins were performed by aligning them to the PF00067.hmm with HMMER package. The consensus logos were generated by Weblogo http://weblogo.threeplusone.com/create.cgi.

Fig. S5 The gene structure of P450 genes. a The gene structure of P450 genes from brown algae. b The gene structure of P450 genes from diatoms. $\mathbf{c}$ The boxplot showing the intron count distribution.

Fig. S6 Chromosome or scaffold distribution of P450s in brown algae and diatom. The position of each P450 was mapped to the genome. Es: E. siliculosus, $\mathrm{Sj}: S$. japonica, Co: $C$. okamuranus, Pt: Phaeodactylum tricornutum, Tp: Thalassiosira pseudonana

Table S1 Statistics of P450 number in each species.

Table S2 Results of BLAST against 1KP transcriptome database using CYPs only occurring in brown algae (CYP5161, CYP5162, CYP5163 and CYP5164) and CYPs only occurring in diatoms (CYP5021, CYP5022 and CYP5165)

Table S3 CYP list, showing the CYP name, protein length, subcellular location, and transmembrane helix number (TH).

Table S4 Sequence identity between brown algal CYP51.

Table S5 Statistics of Codeml calculation.

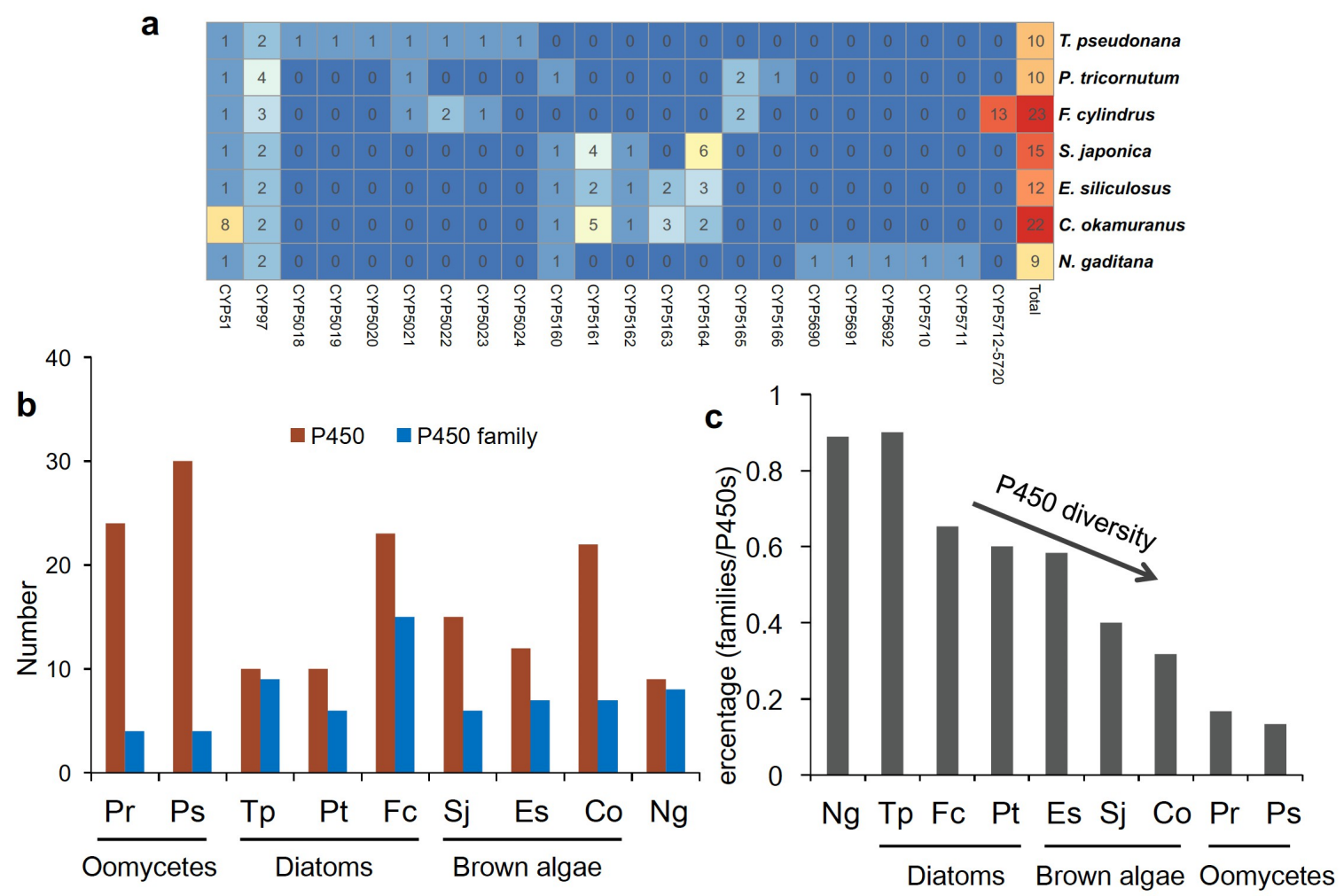

fig.1 


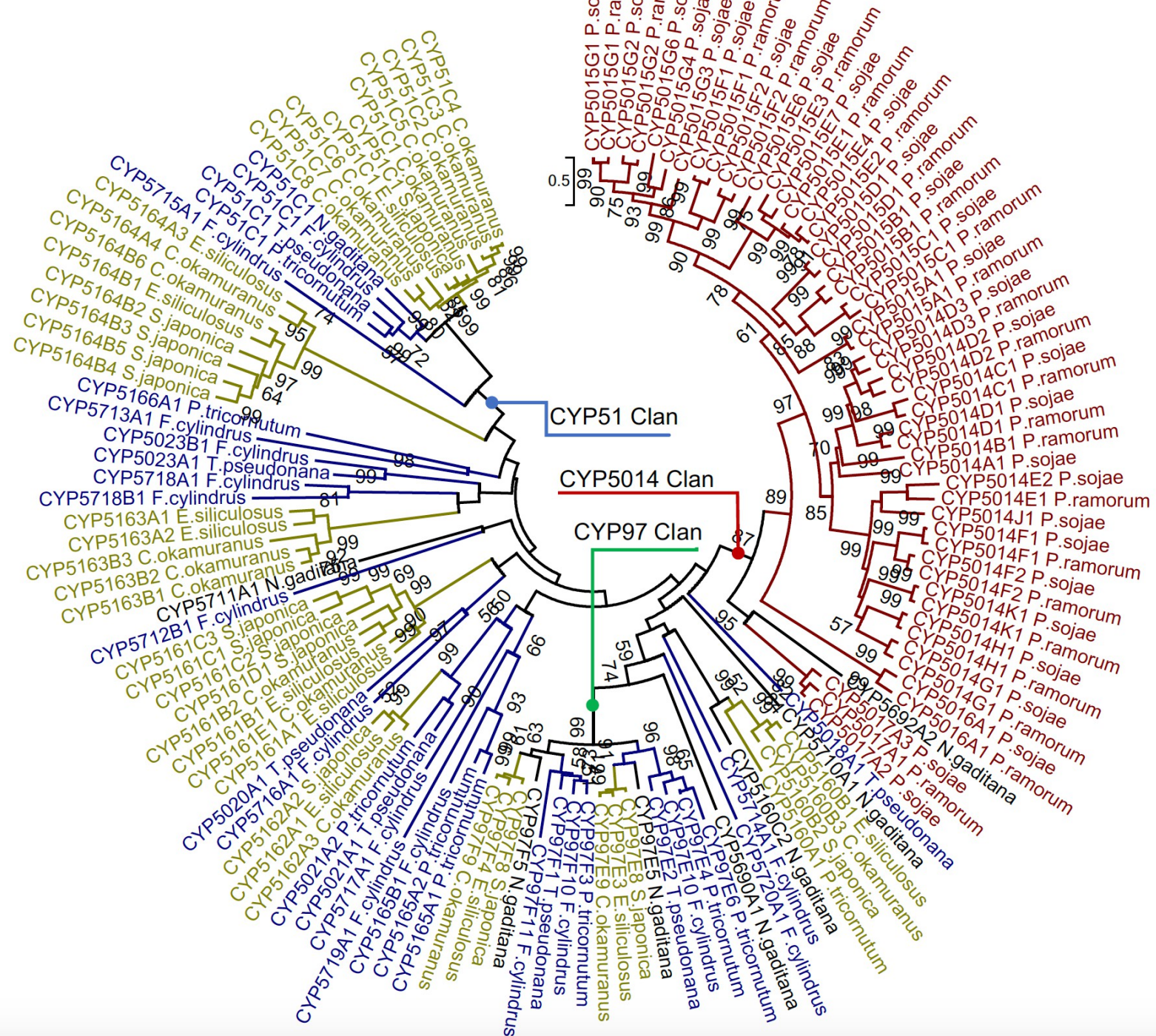

fig. 2

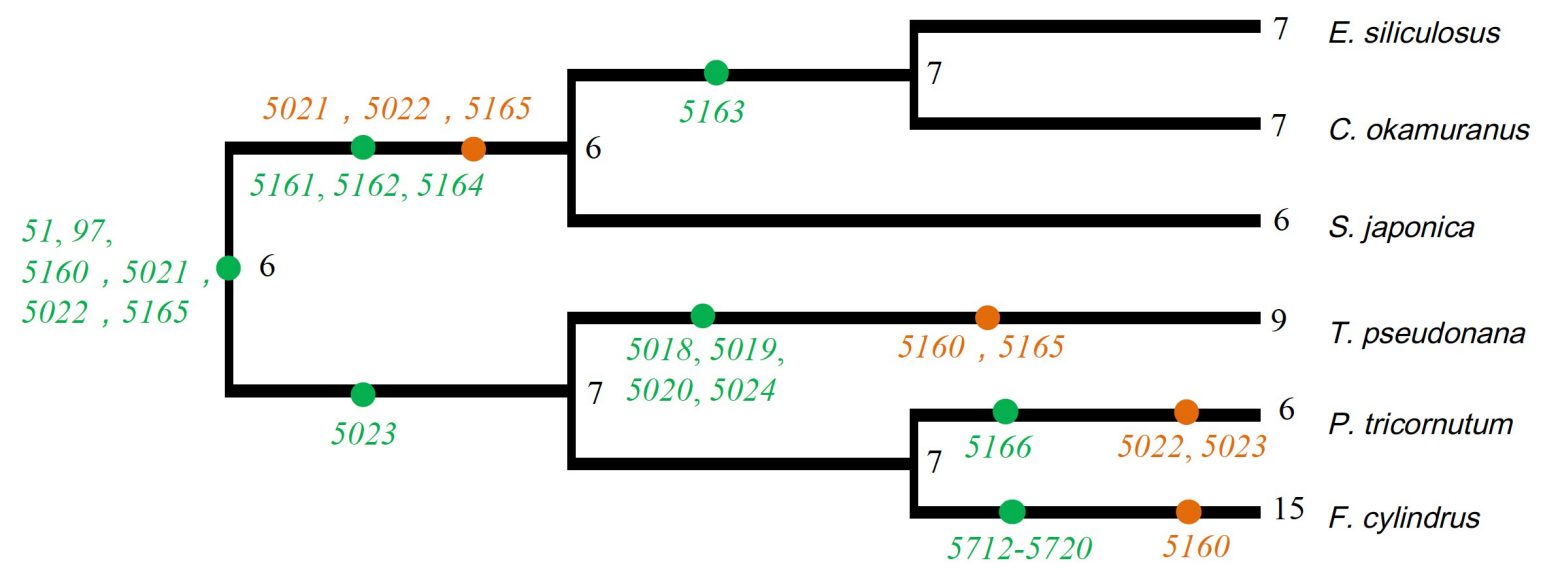

fig. 3 
Figure 4
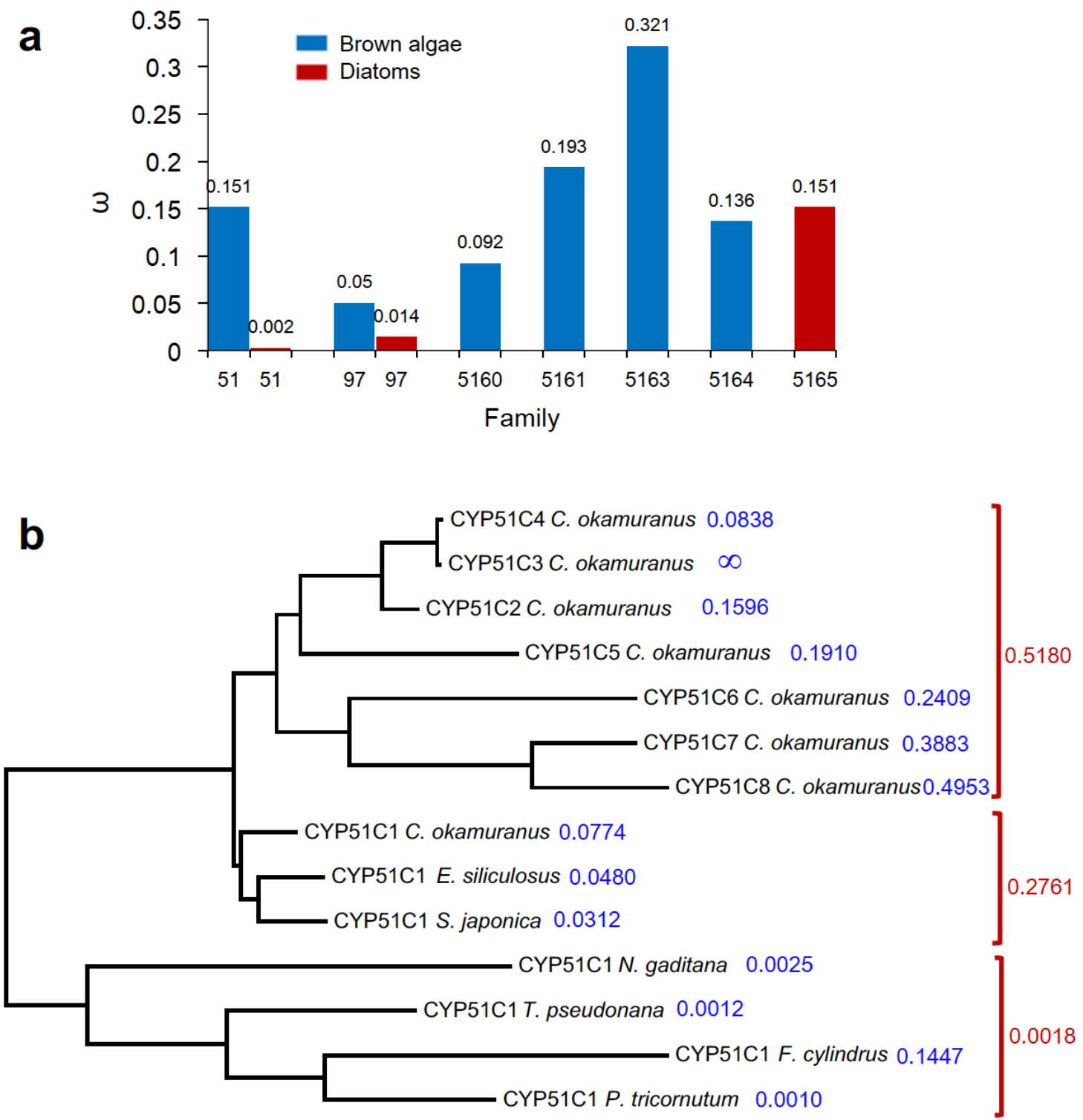

$\longmapsto$ 


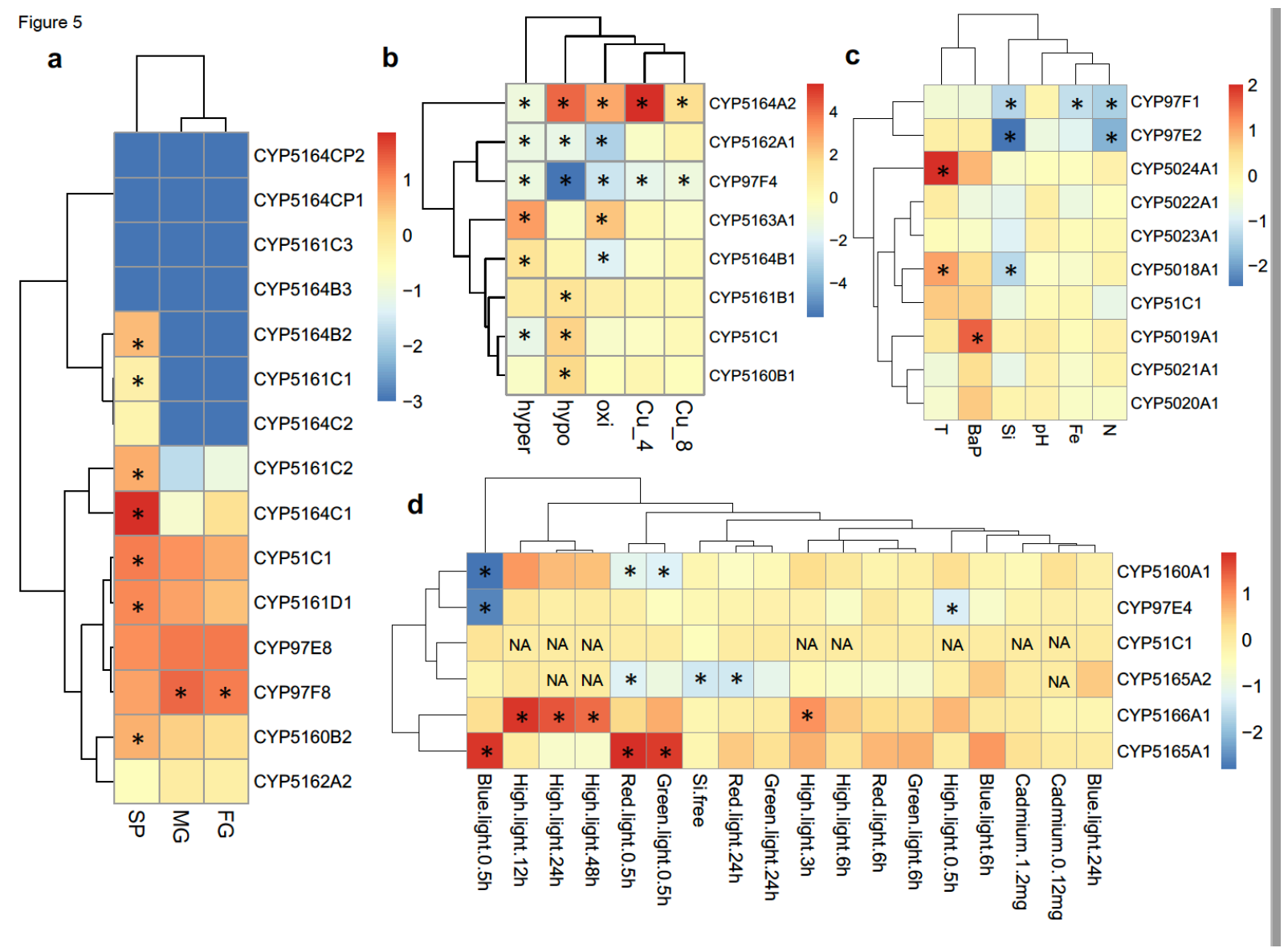




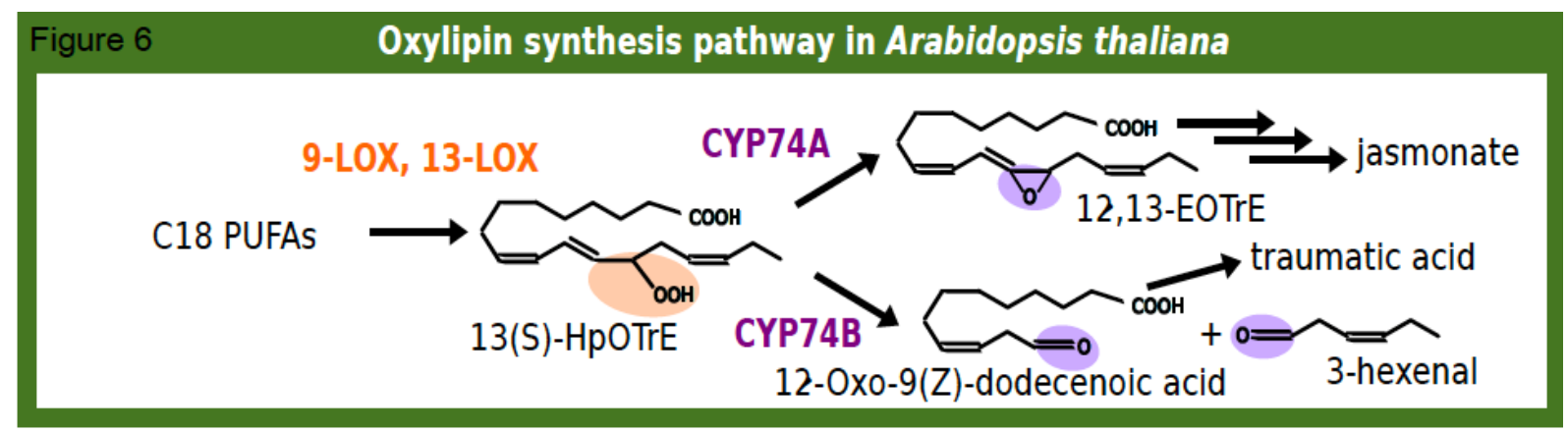

Oxylipin synthesis pathway in Ectocarpus siliculosus

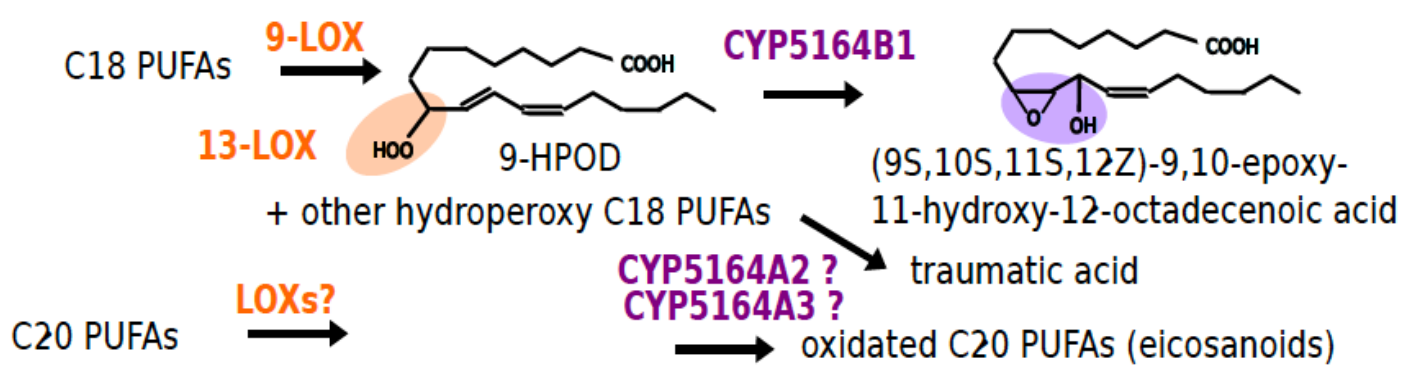

\section{Oxylipin synthesis pathway in Saccharina}

C18 PUFAs $\stackrel{\text { 9-LOX }}{\longrightarrow}$ 9-Hydroperoxy-C18 PUFAs $\longrightarrow$ C6 aldehydes

$$
\begin{aligned}
& \text { CYP5164B2 ? } \\
& \text { CYP5164B3 ? } \\
& \text { CYP5164C2 ? } \\
& \text { CYP5164C3 ? }
\end{aligned}
$$

C20 PUFAs $\stackrel{\text { 12-LOX }}{\underset{15-L 0 X}{12} 15-H y d r o p e r o x y-C 20 \text { PUFAs }} \begin{array}{ll}\text { C6 aldehydes } \\ \text { C9 aldehydes }\end{array}$

\section{Oxylipin synthesis pathway in Thalassiosiria}

C16 PUFAs $\stackrel{\text { 9-LOX }}{\longrightarrow}$ 9-Hydroperoxy- $\longrightarrow$ C8 aldehydes + C16 PUFAs non-P450 HPL Hydroxy-octenoic acids

C20 PUFAs $\stackrel{11 \text {-LOX }}{\longrightarrow} \begin{array}{ll}11-\text { Hydroperoxy- } \\ \text { C20 PUFAs non-P450 HPL }\end{array} \begin{aligned} & \text { C10 aldehydes }+ \\ & 10 \text {-hydroxy-5Z,8Z-decadienoic acid }\end{aligned}$ 


\section{Supplementary material}

Diversity and evolution of cytochromes P450 in Stramenopiles

Linhong Teng ${ }^{1,2}$, Xiao Fan ${ }^{1}$, David R. Nelson ${ }^{3}$, Wentao Han ${ }^{1}$, Xiaowen Zhang ${ }^{1}$, Dong Xu ${ }^{1}$, Hugues Renault $^{4}$, Gabriel V. Markov ${ }^{5}$, Naihao $\mathrm{Ye}^{1,2, *}$

${ }^{1}$ Yellow Sea Fisheries Research Institute, Chinese Academy of Fishery Sciences, Qingdao, China, 266071

${ }^{2}$ Function Laboratory for Marine Fisheries Science and Food Production Processes, Qingdao National Laboratory for Marine Science and Technology, China, 266071

${ }^{3}$ Department of Microbiology, Immunology and Biochemistry, University of Tennessee Health Science Center, 858 Madison Ave. Suite G01, Memphis, TN, USA 38163

${ }^{4}$ Institute of Plant Molecular Biology, CNRS, University of Strasbourg, 67084 Strasbourg, France

${ }^{5}$ Sorbonne Université, CNRS, Integrative Biology of Marine Models (LBI2M), Station Biologique de Roscoff (SBR), 29680 Roscoff, France

*Corresponding author: E-mail, yenh@ysfri.ac.cn; Tel/Fax: +86-532-85830360 

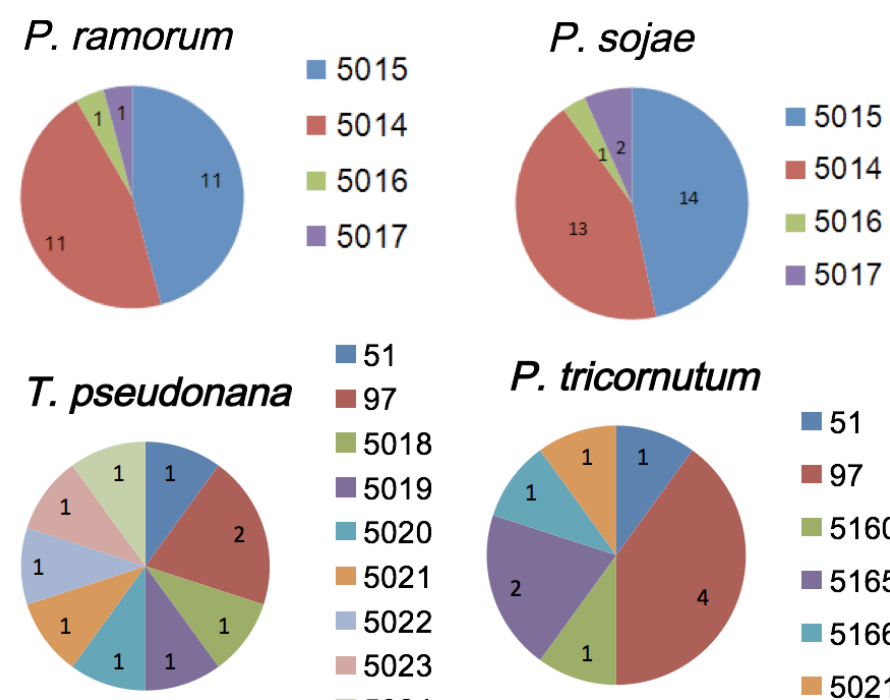

$\square 1$
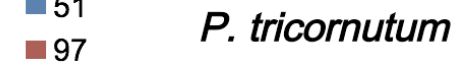

5018

-5019

$\square 020$

5021

$-5022$

5023

P. tricornutum

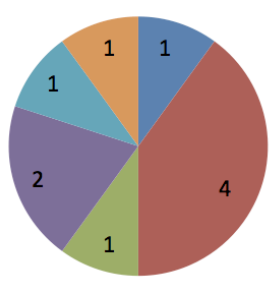

51

- 97

5160

5165

5166

5024

S. japonica

$$
\begin{aligned}
& \square 1 \\
& \square 97 \\
& \square 5160 \\
& \square 5161 \\
& \square 5162 \\
& \square 5164
\end{aligned}
$$

\section{E. siliculosus}

5021
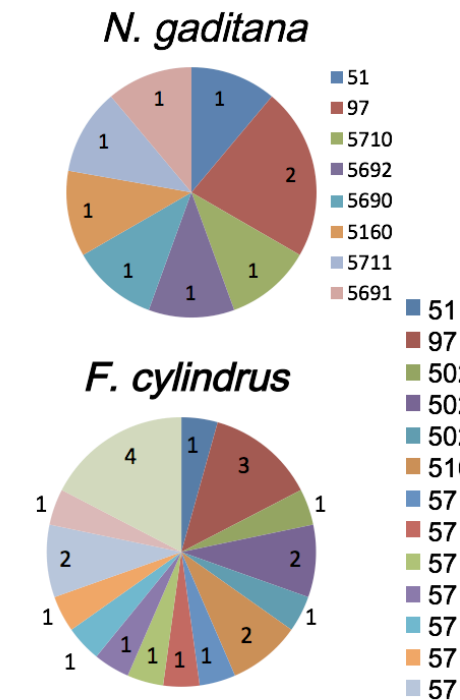

97
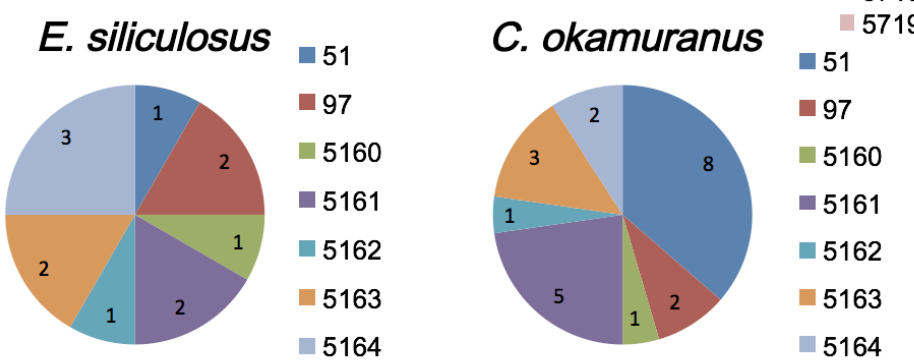

Fig. S1 Statistics of P450 families in each stramenopiles species. 


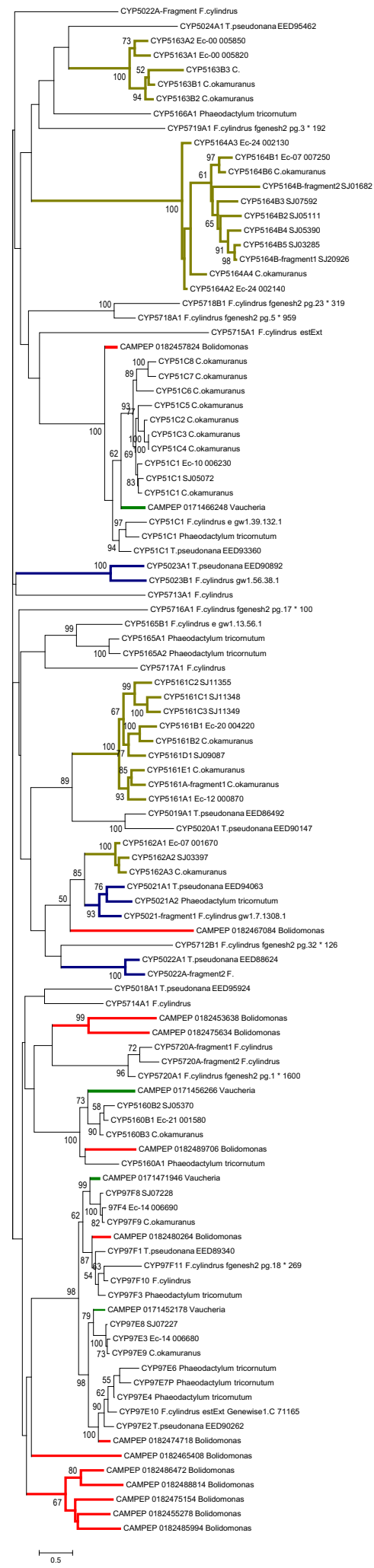

Fig. S2 Phylogenetic tree of CYPs in brown algae, diatoms, Bolidomonas pacifica and Vaucheria litorea. The ML tree was constructed using MEGA 7.0 with WAG+G model. The dark yellow branches are CYPs only occurring in brown algae. The blue branches are CYPs only occurring in diatoms. The red and green branches correspond to CYPs from Bolidomonas and Vaucheria, respectively. 


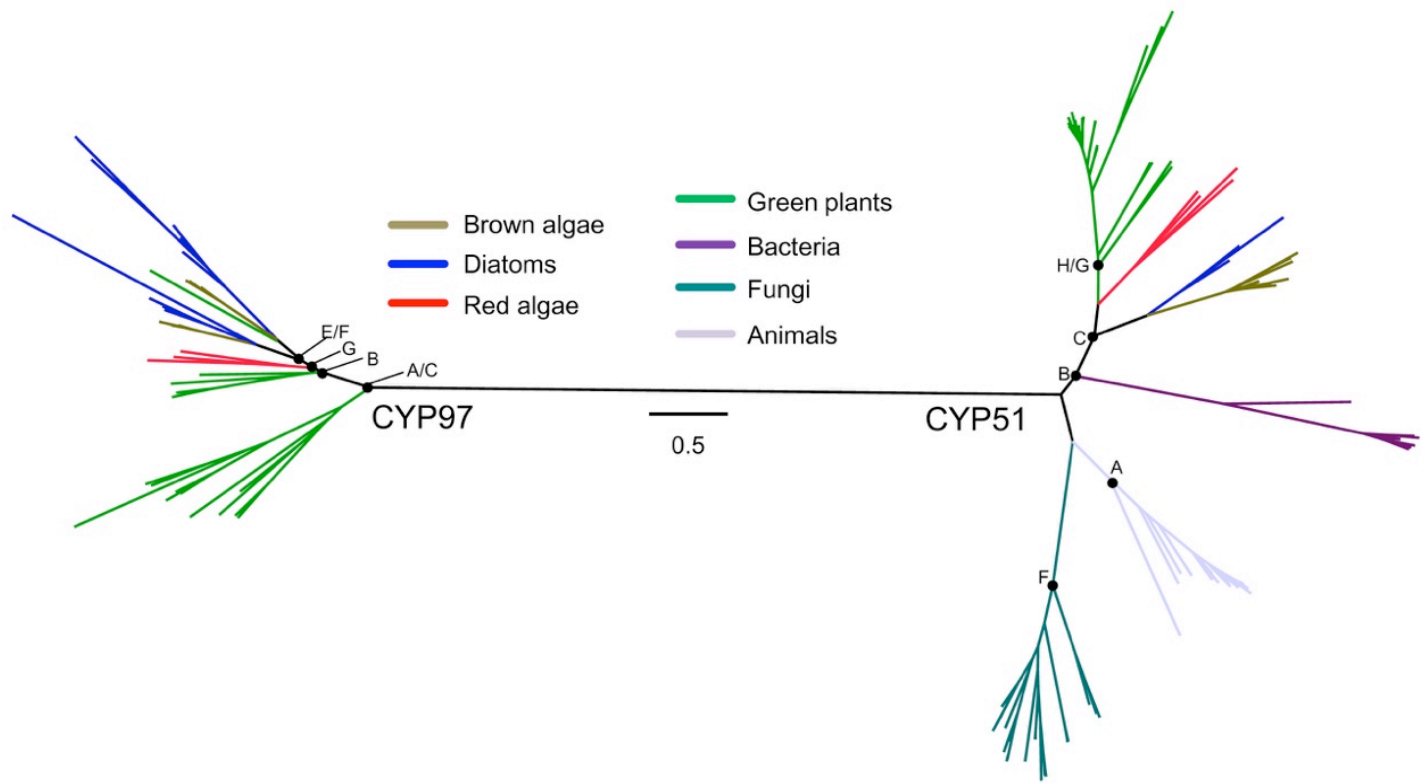

Fig. S3 Phylogeny of brown algae and diatoms CYP51 and CYP97 with their counterparts in animal, plant, bacterial and fungi. The phylogenetic tree was constructed with MEGA 7 and displayed with Figtree, using the alignments of CYPs generated by hmmalign in HMMER program with CYP sequences and PF00067.hmm as input files. 16 animal CYP51s, 10 bacteria CYP51s, 27 plant CYP51s, 21 fungal CYP51s and 16 plant CYP97s were extracted from the CYP homepage http://drnelson.uthsc.edu/CytochromeP450.html.

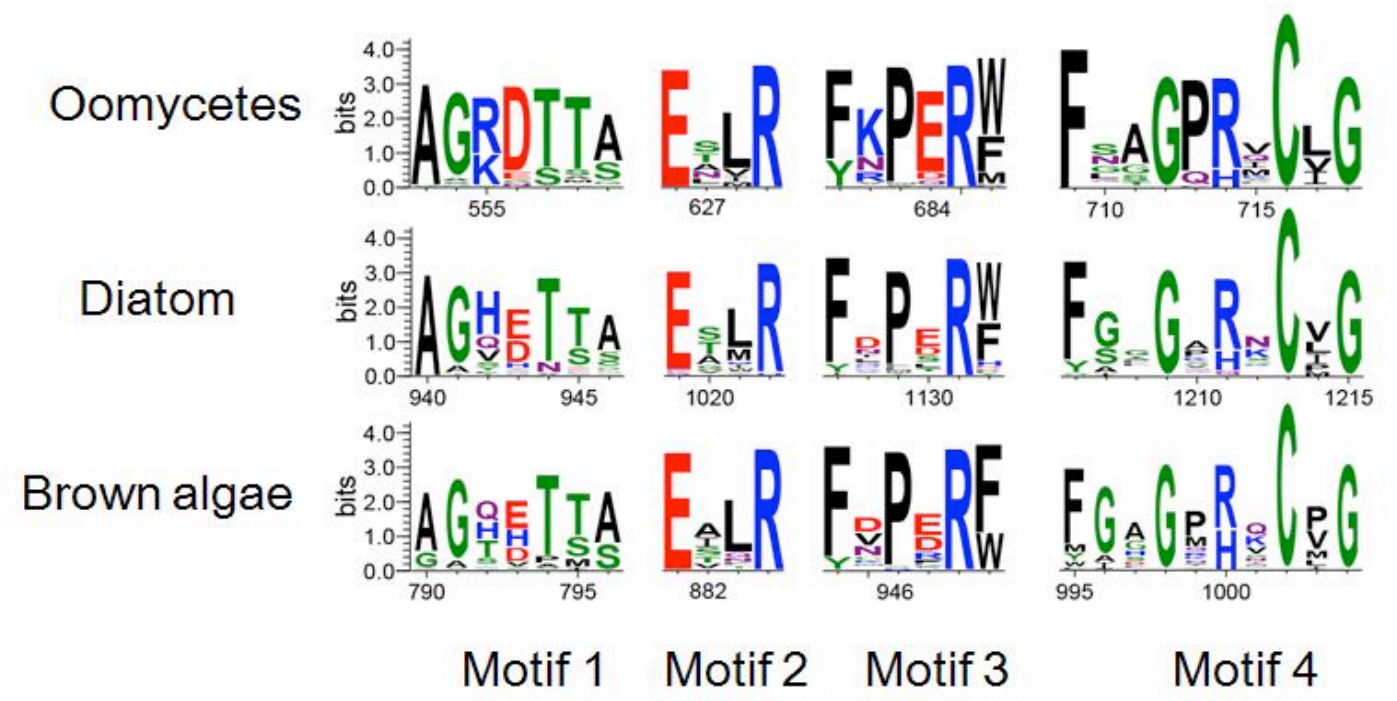

Fig. S4 Sequence logos of the conserved CYP motifs from the three lineages of oomycetes, diatoms and brown algae. Multiple alignments of CYP proteins were performed by aligning them to the PF00067.hmm with HMMER package. The consensus logos were generated by Weblogo http://weblogo.threeplusone.com/create.cgi. 

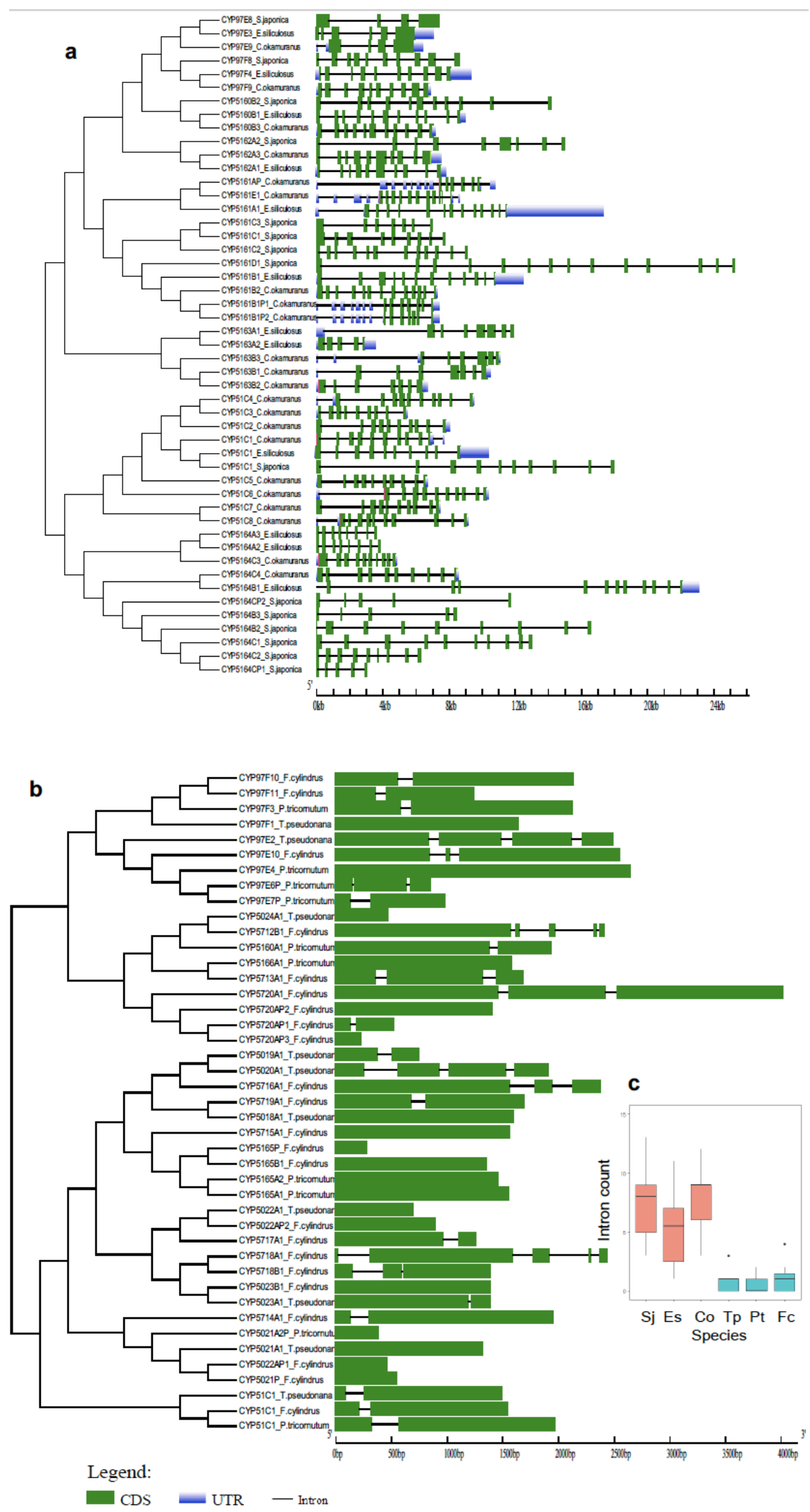

Fig. S5 The gene structure of P450 genes. a The gene structure of P450 genes from brown algae. b The gene structure of P450 genes from diatoms. $\mathbf{c}$ The boxplot showing the intron count distribution. 

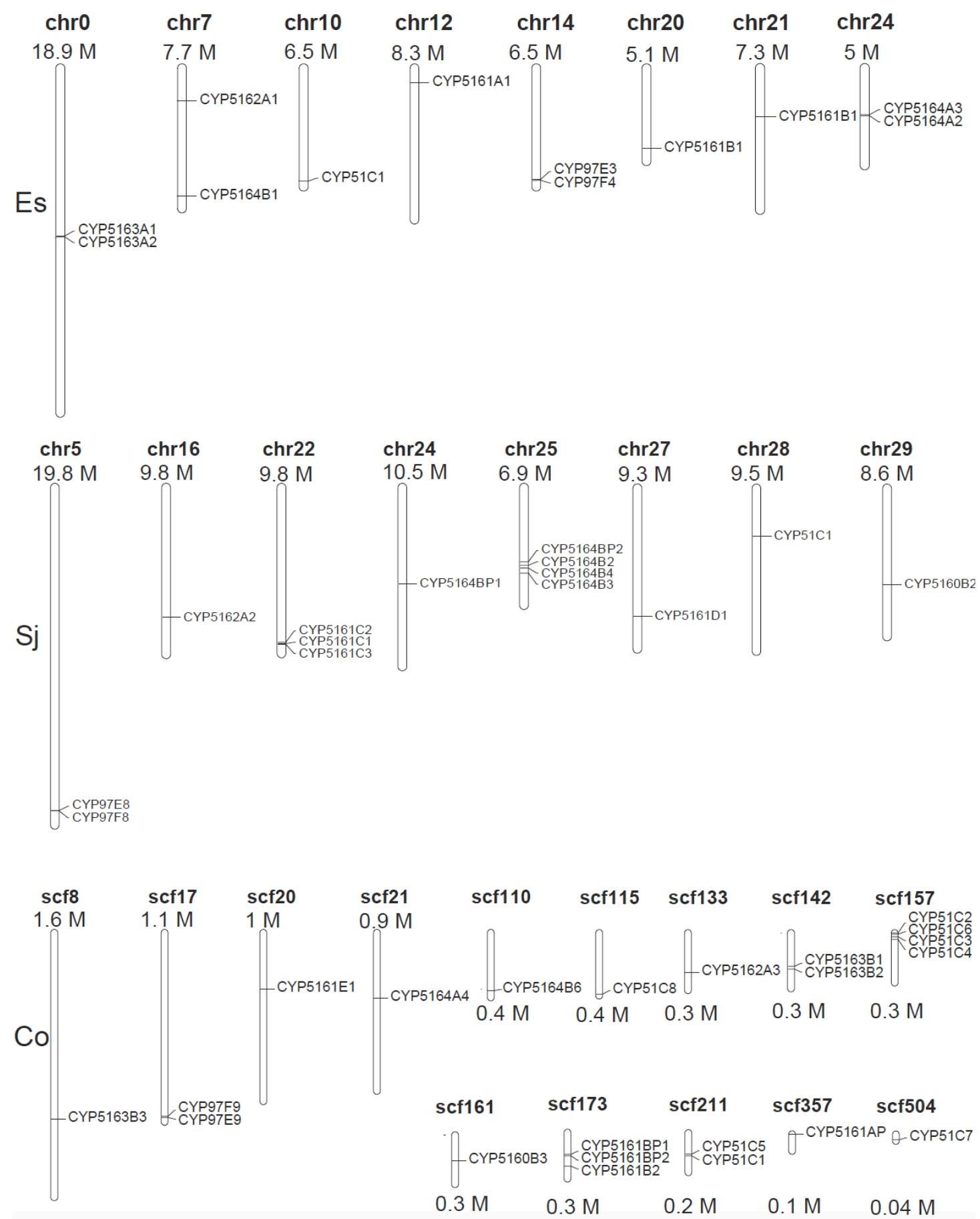


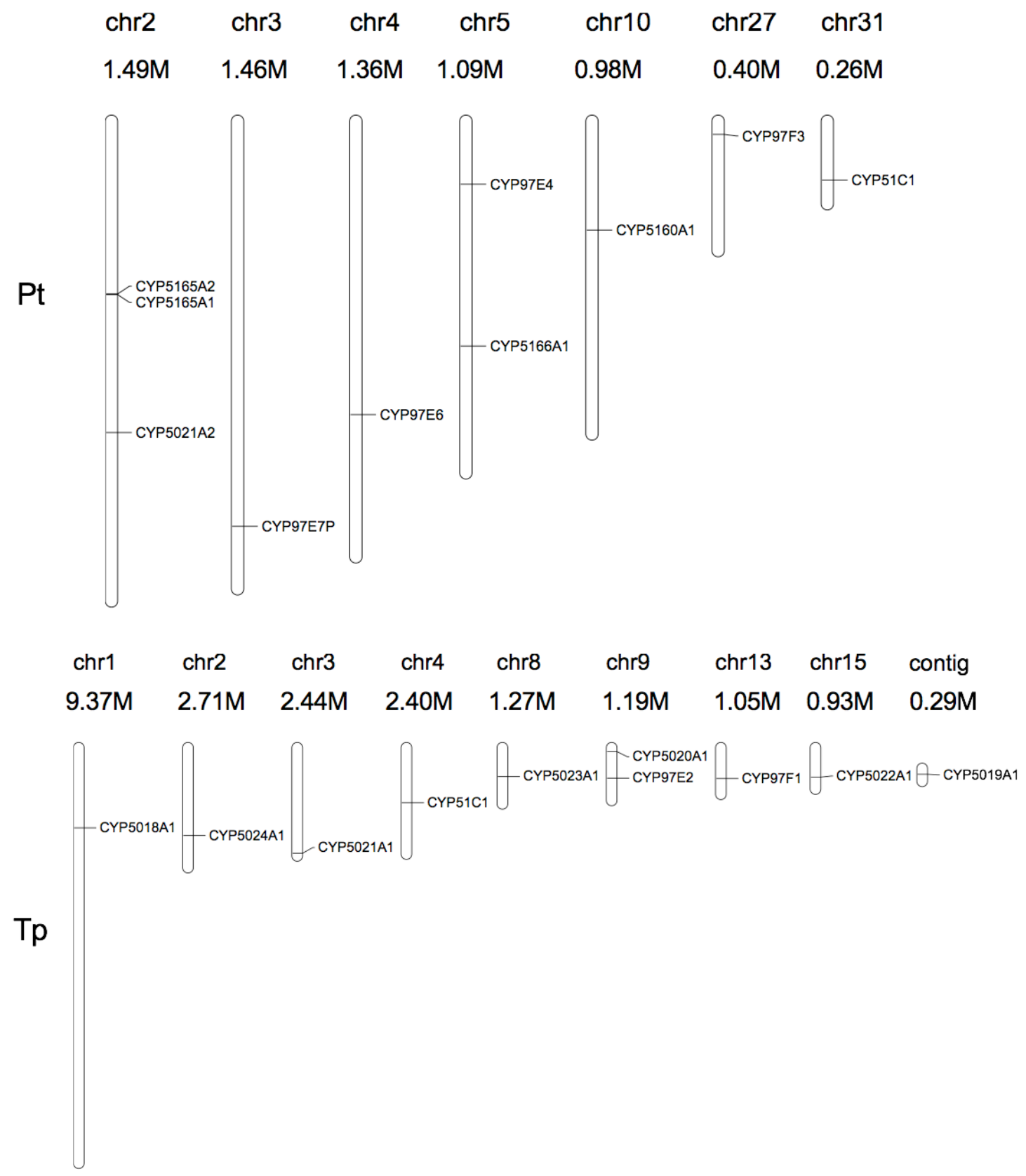

Fig. S6 Chromosome distribution of P450s in brown algae and diatoms. The position of each P450 was mapped to the genome. Es: E. siliculosus, $\mathrm{Sj}: S$. japonica, Co: $C$. okamuranus, Pt: Phaeodactylum tricornutum, Tp: Thalassiosira pseudonana

Table S1 Statistics of P450 number in each species.

\begin{tabular}{lccc}
\hline Species name & No. of P450s & No. of P450 families & No. of P450 subfamilies \\
\hline Phytophthora ramorum & 24 & 4 & 17 \\
Phytophthora sojae & 30 & 4 & 18 \\
Thalassiosira pseudonana & 10 & 9 & 10 \\
Phaeodactylum tricornutum & 10 & 6 & 7 \\
Fragilariopsis cylindrus & 23 & 15 & 17 \\
Saccharina japonica & 15 & 6 & 10 \\
Ectocarpus siliculosus & 12 & 7 & 10 \\
Cladosiphon okamuranus & 22 & 7 & 9 \\
Nannochloropsis gaditana & 9 & 8 & \\
\hline
\end{tabular}


Table S2 Results of BLAST against 1KP transcriptome database (https://db.cngb.org/onekp) using CYPs only occurring in brown algae (CYP5161, CYP5162, CYP5163 and CYP5164) and CYPs only occurring in diatoms (CYP5021, CYP5022, CYP5023 and CYP5165).

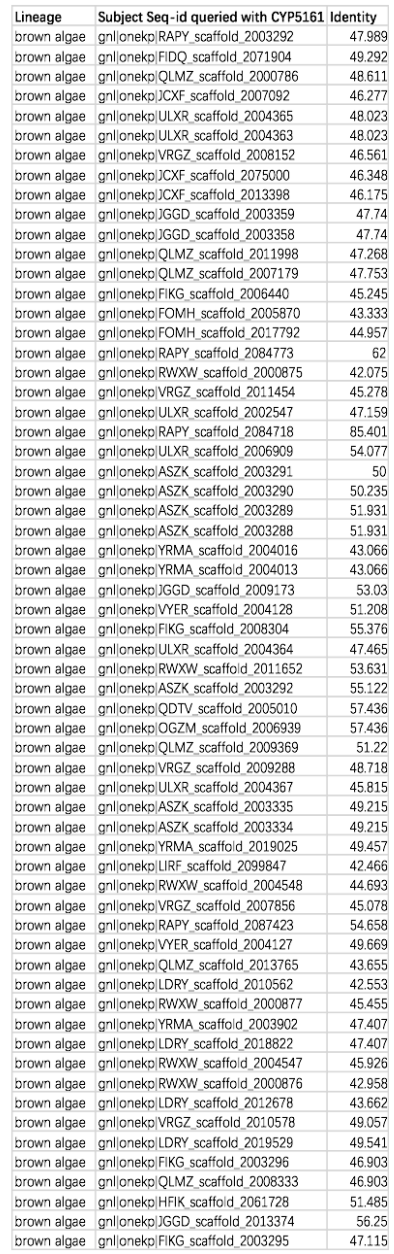

\begin{tabular}{|c|c|c|}
\hline Lineage & Subject Seq-id queried with CYP5162 & Identity \\
\hline brown algae & gnllonekp|FSQE_scaffold_2048840 & 86.905 \\
\hline rown algae & gnllonekp|JCXF_scaffold_2002771 & 75.13 \\
\hline brown algae & gnllonekp|LIRF_scaffold_2013308 & 75.449 \\
\hline own algae & gn|lonekp|VYER_scaffold_2015145 & 71.667 \\
\hline bwn algae & gnllonekp|RWXW_scaffold_2007060 & 74.556 \\
\hline own algae & gn|lonekp|YRMA_scaffold_2103644 & 71.667 \\
\hline own algae & gn|lonekp|F|KG_scaffolc & 75.301 \\
\hline own algae & gn|lonekp|QLMZ_scaffold_2013683 & 74.126 \\
\hline rown algae & gnil|onekp|FOMH_scaffold_2015399 & 74.809 \\
\hline own algae & gnllonekp|HFIK_scaffe & 72 \\
\hline own algae & gnllonekp|JGGD_scaff & \\
\hline own algae & d_2081428 & 83.178 \\
\hline brown algae & gnil|onekp|ULXR_scaffold_2061008 & \\
\hline & & \\
\hline own algae & gnilonekp|VRGZ_scaffold_2020794 & 56.355 \\
\hline brown algae & gn|lonekp|QLMZ_scaffold_2006153 & \\
\hline brown algae & gn|lonekp|RWXW_scaffold_2008007 & 43.478 \\
\hline brown algae & & \\
\hline brown algae & & \\
\hline brown algae & & 175 \\
\hline brown algae & gn|lonekp|VYER_sca & 41.495 \\
\hline brown algae & & 43.048 \\
\hline brown als & & \\
\hline brown algae & gnl|onekp|LDRY_sce & 2.276 \\
\hline wn algae & & \\
\hline brown algae & gn|lonekp|RWXW_scaffold_2 & 1.139 \\
\hline brown alaae & & \\
\hline brown algae & gnllo & 3.201 \\
\hline brown algae & gnllon & 45.872 \\
\hline brown al & & \\
\hline brown algae & gnllone & 43.111 \\
\hline ont & & 451 \\
\hline brown algae & gnilon & 0.543 \\
\hline brown algae & & \\
\hline brown alg. & & 7.895 \\
\hline brown & & \\
\hline & & \\
\hline brown algae & gnllor & 46.701 \\
\hline brown algae & & \\
\hline brown algae & & 439 \\
\hline brown al & & \\
\hline brown algae & & .867 \\
\hline & & 57.282 \\
\hline brown algae & & 55.34 \\
\hline & & 48.819 \\
\hline brown alga & & \\
\hline & & 50 \\
\hline & gnllonekp|FSQE_scaffold_2044923 & \\
\hline & & \\
\hline & & \\
\hline & & \\
\hline & & \\
\hline
\end{tabular}

Lineage Subject Seq-id queried with CYP5021 Identity \begin{tabular}{|l|l|l|l|}
\hline brown algae & gnl|onekp|FSQE scaffold 2048840 & 47.312 \\
\hline
\end{tabular} brown algae gnllonekp|LIRF_scaffold_2013308 46.597 \begin{tabular}{|l|l|l|}
\hline brown algae gnllonekp|JCXF_scaffold_2002771 & 41.204 \\
\hline
\end{tabular} \begin{tabular}{|l|l|l|} 
brown algae gnl|onekp|VYER_scaffold_2015145 & 43.478 \\
\hline
\end{tabular} brown algae gnllonekp|RWXW_scaffold_2007060 43.243 brown algae gnllonekp|YRMA_scaffold_2103644 43.478 brown algae gnl|onekp|FIKG_scaffold_2015075 44.505 brown algae gnl|onekp|QLMZ_scaffold_2013683 44.805 brown algae gnl|onekp|FOMH_scaffold_2015399 $\quad 45$ brown algae gnl|onekp|HFIK_scaffold_2063485 $\quad 45$ brown algae gnl|onekp|VRGZ_scaffold_2081428 42.623

Lineage Subject Seq-id queried with CYP5022 Identity $\begin{array}{lll}\text { Cryptophyta gnllonekp||RZA_scaffold_2061631 } & 42.424\end{array}$

Lineage Subject Seq-id queried with CYP5165 Identity Chrysophyceae gnllonekp|EBWI_scaffold_2034733 44.586 \begin{tabular}{|l|r|r|}
\hline Haptophyceae & gnl||onekp|NMAK_scaffold_2038424 & 43.75 \\
\hline Haptophyceae & gnl|lonekp|LLEN_scaffold_2044944 & 43.75 \\
\hline
\end{tabular}

Lineage $\quad$ Subject Seq-id queried with CYP5023 Identity \begin{tabular}{llr} 
Lineage & Subject Seq-id queried with CYP5023 & Identity \\
\hline Cryptophyta & gnllonekp|ROZZ_scaffold_2062059 & 28.904
\end{tabular}

Table S3 CYP list, showing the CYP name, protein length, subcellular location, and transmembrane helix number $(\mathrm{TH})$

\begin{tabular}{lccc}
\hline CYP name & Protein length & Subcellular location & TH \\
\hline Thalassiosira pseudonana & & & 0 \\
51C1 & 446 & ER & 0 \\
97E2 & 736 & Plastid & 0 \\
97F1 & 546 & ER & 0 \\
5018A1 & 537 & ER & 0 \\
5019A1 & 625 & Cytoplasm, ER, Nucleus & 2 \\
5020A1 & 463 & ER & 0 \\
5021A1 & 418 & ER , Mitochondrion & 0 \\
5022A1 & 488 & ER & 0 \\
5023A1 & 457 & ER & 0 \\
5024A1 & 530 & ER & 0 \\
Fragilariopsis cylindrus & & & 0 \\
5717A1 & 374 & ER & 0 \\
97F10 & 627 & Plastid & 0 \\
5165B1 & 453 & ER & \\
5022AP2 & 299 & ER & \\
\hline
\end{tabular}




\begin{tabular}{|c|c|c|c|}
\hline 5022AP1 & 156 & ER, Mitochondrion & 0 \\
\hline 5720A1 & 1273 & ER & 0 \\
\hline 5718B1 & 392 & ER & 0 \\
\hline $5021 \mathrm{P} 1$ & 183 & ER & 0 \\
\hline 97E10 & 741 & Plastid & 2 \\
\hline 5165P1 & 94 & ER, Mitochondrion & 0 \\
\hline $97 F 11$ & 382 & Cytoplasm, ER & 1 \\
\hline 5718A1 & 517 & ER & 0 \\
\hline 5719A1 & 522 & ER & 1 \\
\hline 5712B1 & 579 & Plastid & 0 \\
\hline $51 \mathrm{C} 1$ & 479 & Plastid & 1 \\
\hline 5716А1 & 655 & Cytoplasm,ER, nucleus & 0 \\
\hline 5720AP1 & 158 & Cytoplasm, ER & 0 \\
\hline 5720AP3 & 76 & ER & 0 \\
\hline 5023B1 & 463 & ER & 0 \\
\hline 5720AP2 & 144 & ER & 0 \\
\hline 5714A1 & 597 & ER & 0 \\
\hline 5715A1 & 520 & ER & 2 \\
\hline 5713A1 & 488 & ER & 0 \\
\hline \multicolumn{4}{|c|}{ Phaeodactylum tricornutum } \\
\hline $51 \mathrm{C1}$ & 482 & ER & 1 \\
\hline 97F3 & 538 & ER & 0 \\
\hline $5160 A 1$ & 565 & ER & 1 \\
\hline 5166A1 & 528 & ER & 0 \\
\hline 97E4 & 769 & ER & 0 \\
\hline 97E6 & 345 & ER & 0 \\
\hline 97E7P & 266 & ER, Mitochondrion & 0 \\
\hline 5021A2 & 634 & ER & 0 \\
\hline 5165A1 & 488 & ER & 2 \\
\hline 5165A2 & 487 & ER & 1 \\
\hline \multicolumn{4}{|c|}{ Saccharina japonica } \\
\hline $51 C 1$ & 485 & ER & 1 \\
\hline 97E8 & 756 & ER, Mitochondrion & 0 \\
\hline $97 F 8$ & 633 & Plastid & 0 \\
\hline 5160B2 & 571 & ER & 0 \\
\hline $5161 \mathrm{C} 1$ & 471 & ER & 2 \\
\hline $5161 \mathrm{C} 2$ & 485 & ER & 2 \\
\hline $5161 \mathrm{C} 3$ & 348 & ER & 1 \\
\hline 5161D1 & 574 & ER & 0 \\
\hline 5162A2 & 615 & ER, Mitochondrion & 1 \\
\hline 5164B2 & 433 & ER & 0 \\
\hline 5164B3 & 359 & Cytoplasm, ER & 0 \\
\hline 5164B4 & 480 & Cytoplasm, ER & 0 \\
\hline 5164B5 & 382 & Cytoplasm, ER & 0 \\
\hline 5164BP1 & 212 & ER & 0 \\
\hline 5164BP2 & 173 & Mitochondrion & 0 \\
\hline \multicolumn{4}{|c|}{ Ectocarpus siliculosus } \\
\hline $5162 A 1$ & 659 & Cytoplasm, ER, Mitochondrion & 2 \\
\hline 5164B1 & 536 & Cytoplasm, ER & 0 \\
\hline $51 C 1$ & 520 & ER & 3 \\
\hline 5161A1 & 530 & ER & 0 \\
\hline 97E3 & 774 & Cytoplasm, ER & 0 \\
\hline 97F4 & 627 & Plastid & 0 \\
\hline 5161B1 & 562 & ER & 1 \\
\hline 5160B1 & 560 & ER & 1 \\
\hline 5164A3 & 300 & ER & 0 \\
\hline 5164A2 & 286 & ER & 0 \\
\hline 5163A2 & 552 & Plastid & 0 \\
\hline 5163A1 & 564 & Plastid & 1 \\
\hline
\end{tabular}




\begin{tabular}{|lccc|}
\hline 5163B3 & 398 & Cytoplasm, ER & 0 \\
\hline 97F9 & 638 & Plastid & 1 \\
\hline 97E9 & 763 & ER & 0 \\
\hline 5161E1 & 345 & ER & 0 \\
\hline 5164A4 & 546 & Cell membrane, ER & 0 \\
\hline 5164B6 & 532 & Plastid & 0 \\
\hline 51C8 & 494 & ER & 1 \\
\hline 5162A3 & 728 & ER, Mitochondrion & 2 \\
\hline 5163B1 & 527 & ER & 0 \\
\hline 5163B2 & 463 & Plastid & 1 \\
\hline 51C2 & 490 & ER & 1 \\
\hline 51C6 & 491 & ER & 1 \\
\hline 51C3 & 414 & ER & 0 \\
\hline 51C4 & 490 & ER & 1 \\
\hline 5160B3 & 558 & ER & 0 \\
\hline 5161BP1 & 229 & ER & 0 \\
\hline 5161BP2 & 229 & ER & 0 \\
\hline 5161B2 & 514 & ER & 0 \\
\hline 51C5 & 492 & ER & 1 \\
\hline 51C1 & 483 & ER & 2 \\
\hline 5161AP1 & 214 & ER & 0 \\
\hline 51C7 & 492 & ER & 1 \\
\hline Nannochloropsis gaditana & & & \\
\hline 97F5 & 540 & ER & 0 \\
\hline CYP5710A1 & 1221 & ER & 0 \\
\hline CYP5692A2 & 527 & ER & 1 \\
\hline 51C1 & 507 & ER & 0 \\
\hline CYP5690A1 & 518 & ER \\
\hline CYP5160C2 & 579 & ER & 1 \\
\hline CYP5711A1 & 375 & ER \\
\hline CYP97E5 & 699 & ER & 0 \\
\hline CYP5691A2P & 163 & ER & 0 \\
\hline
\end{tabular}

Table S4 Sequence identity between brown algal CYP51.

\begin{tabular}{llllllllll}
\hline & $\mathbf{1}$ & $\mathbf{2}$ & $\mathbf{3}$ & $\mathbf{4}$ & $\mathbf{5}$ & $\mathbf{6}$ & $\mathbf{7}$ & $\mathbf{8}$ & $\mathbf{9}$ \\
\hline 1.CYP51C1_Sj & & & & & & & & & \\
\hline 2.CYP51C1_Es & 0.817 & & & & & & & & \\
3.CYP51C8_Co & 0.621 & 0.588 & & & & & & & \\
\hline 4.CYP51C2_Co & 0.781 & 0.723 & 0.611 & & & & & & \\
5.CYP51C6_Co & 0.649 & 0.618 & 0.582 & 0.641 & & & & \\
6.CYP51C3_Co & 0.651 & 0.616 & 0.51 & 0.761 & 0.547 & & & & \\
7.CYP51C4_Co & 0.757 & 0.698 & 0.603 & 0.889 & 0.645 & 0.838 & & & \\
8.CYP51C5_Co & 0.719 & 0.666 & 0.591 & 0.707 & 0.627 & 0.594 & 0.699 & & \\
9.CYP51C1_Co & 0.86 & 0.813 & 0.619 & 0.787 & 0.653 & 0.671 & 0.761 & 0.731 & \\
\hline 10.CYP51C7_Co & 0.646 & 0.608 & 0.765 & 0.646 & 0.6 & 0.534 & 0.623 & 0.63 & 0.652 \\
\hline
\end{tabular}

Table S5 Statistics of Codeml calculation.

\begin{tabular}{|c|c|c|c|c|c|c|c|}
\hline \multirow[t]{2}{*}{ Family } & \multicolumn{2}{|c|}{ M0 (one-ratio) } & \multicolumn{2}{|c|}{ Site model $-\ln L$} & \multirow[t]{2}{*}{$2 \Delta \ln \mathrm{L}$} & \multirow[t]{2}{*}{ p-value } & \\
\hline & $\omega$ & $-\ln \mathrm{L}$ & M7 & M8 & & & \\
\hline Brown algae CYP51 & 0.1509 & 6911.09 & 6849.41 & 6848.71 & 1.4 & & \\
\hline Diatom CYP51 & 0.00211 & 4126.37 & 4083.87 & 4083.59 & 0.56 & & \\
\hline Brown algae CYP97 & 0.05018 & 8327.44 & 8067.5 & 8046.25 & 42.5 & & $<0.001$ \\
\hline Diatom CYP97 & 0.01406 & 8203.9 & 8055.24 & 8054.17 & 2.14 & & \\
\hline Brown algae CYP5160 & 0.09232 & 4482.55 & 4437.14 & 4433.33 & 7.62 & & $<0.001$ \\
\hline Brown algae CYP5161 & 0.19334 & 4391.33 & 4326.2 & 4326.19 & 0.02 & & \\
\hline Brown algae CYP5163 & 0.32151 & 3669.85 & 3651.38 & 3651.11 & 0.54 & & \\
\hline Brown algae CYP5164 & 0.13675 & 5847.88 & 5772.83 & 5772.82 & 0.02 & & \\
\hline Diatom CYP5165 & 0.15071 & 4402.62 & 4375.29 & 4373.11 & 4.36 & & \\
\hline CYP51 branch site model & $-\operatorname{lnL1}$ & $-\operatorname{lnL} 2$ & $2 \Delta \ln \mathrm{L}$ & & & & \\
\hline Foreground C3-C7 & 10161.6 & 10161.5 & 0.2 & & & & \\
\hline Foreground $\mathrm{C} 3$ & 10163.5 & 10163.4 & 0.2 & & & & \\
\hline
\end{tabular}

